\title{
Number of children and cognitive abilities in later life
}

\author{
Valeria Bordone and Daniela Weber*
}

\begin{abstract}
The investigation on cognition has identified structural characteristics of the social network as important components to contrast cognitive ageing. Using data from the Survey of Health, Ageing and Retirement in Europe, we examine the association between number of children and cognitive functioning in later life, considering the performance in three cognitive tests (fluency, immediate recall and delayed recall). The analyses focus on respondents aged $60+$, not in the labour market, accounting for their proximity and frequency of contact to children. We find a positive association between cognitive functioning and having children: childless men and women name a lower number of items in one test, the so-called "fluency test". However, parents of two children show higher abilities than parents of a single child and heads of large families. Our findings also indicate that more contacts with children are associated with higher fluency. Interestingly, the relationship with the child(ren) is not significantly related to cognitive performance in the two tests of recall abilities, where educational attainment and the presence of a spouse or partner show to be more relevant. Studying both men and women allows us to consider both biological mechanisms and social relationships linking parity and cognitive functioning in later life.
\end{abstract}

\section{Introduction}

Cognitive capacity in the ageing process is of relevance for the individual as well as for thesociety. Cognitive functioning is shown to be a good predictor of health outcomes (Whalley and Deary 2001; Batty et al. 2007) and for mortality (Deary et al. 2005), affecting various aspects of life. The implication of cognitive ageing in

\footnotetext{
* Valeria Bordone (corresponding author), Vienna University of Economics and Business, Wittgenstein Centre for Demography and Global Human Capital, Vienna; International Institute for Applied Systems Analysis (IIASA), Laxenburg; Research Institute Human Capital and Development, Vienna University of Economics and Business, Nordbergstrasse 15/A/6 office 622, 1090 Vienna, Austria. Email: valeria.bordone@wu.ac.at

Daniela Weber, International Institute for Applied Systems Analysis (IIASA), Laxenburg.
}

DOI: 10.1553/populationyearbook2012s095 
the development of mental diseases raises the attention of ageing societies, as it was reflected, for example, in the themes of the 8th World Congress on Active Ageing: prevention and self-management of the conditions associated with cognitive functioning and dementia (http://www.wcaa2012.com). Therefore, researchers and political decision makers in several European countries try to face the challenge of maintaining cognitive abilities later in life (e.g. the symposia "Aktiv Altern - Der österreichische Weg, Bundesplan für Seniorinnen und Senioren" in Austria (http://www.oepia.at), or "Changing images of ageing" in Germany (http://www.dza.de)).

Research concerned with relations between adult age and cognitive functioning has often been based on the distinction, originally formulated by Cattell (1943), between crystallised and fluid abilities. Despite the different patterns of cross-sectional age-cognition relations proposed for different measures of processing (see Salthouse 2010 for a review), both theoretical models and empirical evidence have shown a high plasticity of cognitive functioning and suggest the potential of behaviours and beliefs to maintain cognitive functioning (Baltes and Labouvie 1973; Baltes 1987; Kramer and Willis 2002, 2003; Baltes et al. 2006; Ball et al. 2007; see also Hertzog et al. 2009 for a review).

Other than biological and contextual factors (e.g. Anstey and Smith 1999), education (Le Carret et al. 2003) and being active in the labour market (Bonsang et al. 2012; Mazzonna and Peracchi 2012) may (positively) affect the cognitive endowment. Being involved in leisure and social activities (Fabrigoule et al. 1995; Scarmeas and Stern 2003; Engelhardt et al. 2010), among other behaviours showing social embeddedness, have also been found to significantly contribute to the variation in cognitive ageing, being associated with both the level of cognitive functioning and the rate of age-related change in cognitive functioning (e.g. Bassuk et al. 1999; Fratiglioni et al. 2000; Seeman et al. 2001; Béland et al. 2005). Within the framework of the cognitive enrichment theory (Hertzog et al. 2009), emphasising the potential of behaviour to influence levels of cognitive functioning, and in view of the low fertility rates and increased longevity in Europe, we study the association between cognitive functioning and number of children in eleven European countries, based on the Survey of Health, Ageing and Retirement in Europe (SHARE).

Previous literature has shown a close link between parity and several health outcomes as well as mortality (e.g. Doblhammer 2000; Grundy and Holt 2000; Grundy and Tomassini 2005; see also Kendig et al. 2007 for an extensive review of the different bodies of research that have touched on issues linked with parenthood differences in health). The aim of this study is to extend the knowledge on old-age outcomes, identifying the association between number of children and cognition and adding to it a focus on frequency of contact and geographical distance between parents and adult children. Studying both men and women allows us to consider the association through both biological mechanisms and social relationships in linking parity and later-life cognitive functioning. 
The remaining of the paper is structured as follows: first, we review the existing literature on family size and cognition of older adults; next, we present data and methodology; and then we describe the main statistical findings. In the final sections, after a discussion of alternative interpretations of the results, we conclude and discuss possible future research on the topic.

\section{Family size and cognition in old age: theoretical background and empirical evidence}

\subsection{Cognitive functioning in later life}

The original classification of cognitive dimensions, as formulated by Cattell (1943), distinguished between two main cognitive dimensions: crystallised abilities refer to the knowledge acquired during the life course; fluid abilities consist of the basic mechanisms of processing information carried out at the time of assessment and are closely related to biological and physical factors. Yet, some scholars rather rely on a classification system based on the presumed neuroanatomical locus of task performance (e.g. Glisky et al. 1995). Despite different descriptive terminology, there is consensus on two patterns of crosssectional age-cognition relations: measures representing products of processing carried out in the past (i.e. general knowledge or semantic memory) tend to increase until people are in their $60 \mathrm{~s}$ and then decline; measures representing efficiency or effectiveness of processing carried out at the time of assessment (i.e. working memory) tend to linearly decline from early adulthood (see Salthouse 2010 for a review of cognitive ageing).

Over the last twenty years, much of the cognitive ageing research trying to identify variables that predict cognitive performance in old age has focused on contextual variables and biomarkers: contextual variables refer to mental health and physical activity, meant as environmental influences that create the context in which biological development occurs (e.g. Luszcz et al. 1997); biomarkers are measures of biological ageing, by definition sensitive to age changes (Balin 1994; Anstey et al. 1996). Reports from the unique Swedish Adoption/Twin Study of Aging (SATSA) indicate that genetic influences are important for cognitive abilities (Pedersen et al. 1992; Emery et al. 1998). Medical research has confirmed that genes influence brain function and, at the same time, genetic health problems may stand for nulliparity (O'Donovan and Owen 2009).

Under the umbrella of the cognitive enrichment theory (Hertzog et al. 2009), suggesting that all behaviours and attitudes can potentially affect cognitive performance, cross-sectional, longitudinal and intervention studies have shown that cognitive functioning can be preserved more than has previously been recognised (e.g. Kramer and Willis 2002, 2003; Ball et al. 2007). 
Therefore, understanding the potentials of enhancing cognition and planning the intervention that could protract cognitive decline would be likely to improve older peoples' quality of life (Kramer and Willis 2002) and defer society's costs for long-term care (Hertzog et al. 2009).

Cognitive-training studies, for example, have demonstrated that cognitive functioning in older adults benefits from intensive training in strategies that promote thinking and remembering (e.g. Baltes and Lindenberger 1988; Ball et al. 2002; Baltes et al. 2006; Willis et al. 2006). Similarly, an extensive literature has shown that exercise interventions have substantial benefits for cognitive function, mainly through a reduced risk of diseases associated with cognitive decline (see Hertzog et al. 2009 for a review of these studies).

Population-based studies of adults have explained inter-individual differences in cognitive functioning within age groups also by sociodemographic inequalities. Over and above other factors, education displays a large positive association with later-life cognition in a number of international settings (Cagney and Lauderdale 2002; Lee et al. 2003; Tuong Nguyen et al. 2008; Yount 2008). Such a strong association may stem from various sources, including selection into education, enduring learning during the education process, and education-induced changes in brain function that contribute to maintain higher levels of cognition (Alley et al. 2007). Some of the benefits of education on later-life cognition may additionally be mediated through related life course resources, such as occupation and lifestyle behaviours (Christensen et al. 1996; Tuong Nguyen et al. 2008; Finkel et al. 2009), therefore accounting for part of the contextual variables. In view of our research question, we note that the more highly educated have fewer children (Skirbekk 2008).

In this context, wide evidence also shows that a diverse array of intellectually stimulating activities predicts better maintenance of cognitive functioning and is associated with a reduced risk of developing Alzheimer's disease in late life (see Scarmeas and Stern 2003 for a review of studies that have investigated the association between level of participation in activities and performance on various cognitive tasks): Bonsang and colleagues (2012) and Mazzonna and Peracchi (2012) show that retirement leads to a decline of both fluency and recall abilities (i.e. the two types of cognitive performance considered also in this study and further described in Sections 2.2 and 3); Engelhardt and colleagues (2010) find a positive association between social activities and cognitive endowment when measuring delayed recall; Jopp and Hertzog (2007) show that engaging in activities is correlated with performing at high levels of memory and five other ability factors, independent of age; while Fabrigoule and colleagues (1995) record higher incident dementia on follow-up visits to initially non-demented individuals who were less involved in social and leisure activities. Social exchanges, broadly defined, are recognised to be positively related to better cognitive functioning (e.g. Seeman et al. 2001; Béland et al. 2005). Among the explanatory mechanisms that have been considered, physiological factors affecting the central nervous 
system may be exacerbated for those with lower social support (Berkman et al. 2000).

Although socialisation processes occur within multiple domains over the life course (e.g., school, friends, neighbourhood, workplace), the family shapes the most, and in old age usually becomes the immediate social environment (e.g. Antonucci 1990). Intergenerational family ties attract our attention because they tend to involve a combination of supportive and stressful experiences, especially in later life when the parent-child relationship undergoes a role reversal (Silverstein et al. 1996).

\subsection{Family size and health-related outcomes}

Childbearing and childrearing are widely recognised to encompass both positive emotional outcomes and challenges. The theory of disposable soma (Kirkwood and Rose 1991) posits a trade-off between physical resources used for reproduction and those needed for survival. Previous studies focusing on parity have investigated on its relation with longevity and several health-related outcomes. Doblhammer (2000) describes a U-shaped relationship among English, Welsh and Austrian, with both childless and high-parity women experiencing higher mortality than women with one or two children. Grundy and colleagues (Grundy and Holt 2000; Grundy and Tomassini 2005) find a hump-shaped relation between family size and health among English and Welsh women aged 50+ and born 1911-1940: those without and those with five or more children showed worse health and higher mortality. Buber and Engelhardt (2008), using European SHARE data, show that up to parity three the number of children has a protective effect on elderly persons' mental health (i.e. depression). Kendig and colleagues (2007, p. 1480) find parenthood effects pertaining to health behaviours: both in Finland and in the Netherlands, "having adult children means that people refrain from health-compromising behaviours" such as smoking, alcohol consumption and engage more in physical exercise as compared to their childless counterparts. A negative relation holds between number of children and life satisfaction among people aged 75+ in Germany (Gwozdz and Sousa-Poza 2010). According to Chaves et al. (2009), the number of living children is also a risk factor in developing countries; while more confidants increase the likelihood of "ageing successfully". However, their definition did not consider cognitive impairment nor did they control for curvilinear associations.

The few studies on the effect of reproductive factors on cognition, mainly focusing on non-demented postmenopausal women, have suggested that reproductive events may be associated with enhanced cognition because of higher lifelong estrogen exposure (e.g. Nappi et al. 1999 on attentive and verbal memory performances; Smith et al. 1999 on two verbal factors). Still, evidence remains mixed. In particular, when considering the number of children, some studies found no correlation between parity and cognitive functioning (e.g. Smith et al. 
1999); while in others nulliparity was found to be associated with less cognitive decline in women, suggesting that in addition to affecting a woman's risk for agerelated diseases as osteoporosis and breast cancer, certain reproductive events may influence age-related changes in the central nervous system (McLay et al. 2003 on mini-mental state examination [MMSE] scores).

We therefore wonder if and to what extent there is an association between parents' cognition and the variation in the number of children. This helps us to bring forward a more specific hypothesis on how the number of children should relate to cognitive functioning, investigating a link that is both a function of underlying biological mechanisms and of social relationships. From the literature discussed above, we expect a curvilinear relationship between number of children and cognitive performance of the elderly parents: both nulliparity and high parity are expected to be negatively associated with cognitive performance in later life.

The following analysis is concentrated on two aspects of cognitive ability: fluency and recall. This latter is measured with two tests, immediate and delayed recall. Fluency is usually considered as a measure of efficiency because it uses the ability to keep attention on a given task; recall tests involve both a timing aspect and a processing-speed component. Therefore, these different measures of cognitive functioning are often identified as belonging to the domains of crystallised and fluid abilities, respectively. A measure of crystallised abilities would not involve any processing of new information. The decline of cognitive functioning was found most pronounced for recall by Engelhardt and colleagues (2010) on the same data, suggesting that the first and the other two tests are targeting different cognitive abilities.In this study, we investigate on the association between number of children and cognitive performance, with a special focus on two relevant dimensions of intergenerational solidarity: structure and association. Structural solidarity is the 'opportunity structure' for crossgenerational interaction and reflects geographic proximity between family members; associational solidarity refers to their frequency of contact (Bengtson and Roberts 1991).

To do this, we carry out separate analyses for women and men. The association between social ties and (mental) health is likely to differ by gender (Wethington et al. 1987). Summarising gender differences found in the context of intergenerational relationships, women have been observed to maintain more emotionally intimate relationships than men with children (Fischer 1982; Gerstel and Gallagher 1993). Moreover, a long tradition of research in gerontology has shown that the implications of family size for health are not purely biological and they are therefore not limited to women: while women's exposure to the physiological impact of childbearing and childrearing is more evident, psychological, behavioural and socioeconomic consequences affect both men and women (e.g. Doblhammer and Oeppen 2003; Grundy and Kravdal 2008). 


\section{Data and method}

The present study explores the association between cognitive performance of older adults and the number of children they have, focusing on the structural and associational dimensions of their relationship.

The Survey of Health, Ageing and Retirement in Europe (SHARE) serves the purpose perfectly. This multidisciplinary and cross-national database of micro data on health, socio-economic status, and social and family networks is representative of the non-institutionalised population aged 50 or over in eleven European countries: Austria, Belgium, Denmark, France, Germany, Greece, Italy, the Netherlands, Sweden, Switzerland and Spain (Börsch-Supan et al. 2005). Despite the longitudinal design of SHARE, we use its first wave (conducted in 2004) to overcome any retest effect on the cognitive tests (Schaie and Hofer 2001 discuss this drawback of longitudinal studies of cognition; see Mazzonna and Peracchi 2012 on retest effects in SHARE) and selective attrition, which is a serious issue in panel surveys on elderly (Zamarro et al. (2008) find that in SHARE people in poor health and with poor cognitive abilities are more likely to drop out of the panel). Moreover, to the end of this study, the availability of SHARE's two waves, two years apart, would not significantly improve the information on life cycle cognitive changes related to fertility behaviours or to changes in parent-child relationships.

Following the approach adopted by previous studies on this dataset (e.g. Buber and Engelhardt 2008), we do not consider respondents who were employed or unemployed at the time of interview (i.e. pensioners, homemakers and permanently sick or disabled are included). The interrelation between employment and cognition could not be fully investigated with cross-sectional data: while cognitive ability is a determinant of intellectually demanding work, work complexity is also beneficial to cognitive function (with regard to work itself, see for example the longitudinal study of Hauser and Roan 2007). For the same reason, we only consider people aged at least 60 years old. The sample comprises 10,756 persons (4,778 men and 5,978 women).

The early results in research on cognition indicate that different cognitive abilities exhibit different age trends. We operationalise fluency and recall functioning by three cognitive tests. To measure fluency in SHARE, the interviewer asks the respondent to name as many different animals as he/she can think of, within one minute. Valid answers are any members of the animal kingdom. For immediate recall the interviewer reads ten words and the interviewee has one minute to recall them in any order. After approximately 5 minutes, the respondent faces the delayed recall task: recalling (within one minute) as many words as possible of those heard for the immediate recall test. We acknowledge that the latter is not purely a measure of memory because having previously recalled the same list of words influences the performance on the later test. Moreover, all the three measures are, to some extent, influenced by 
working memory capability (see Oberauer et al. 2000 for a discussion on the facets of working memory capacity).

In order to study the association between children and cognitive performance, we conduct multivariate linear regressions to model cognitive functioning. Regressions are performed separately for men and women to account for different gender-specific effects. Our models focus first on the number of children, distinguishing between no children, one, two, three, four children or more. ${ }^{1}$ Secondly, we construct a variable with value 0 for childless respondents and five categories taking into account the geographical proximity of parents to their (nearest) child (same household or building; less than $1 \mathrm{~km} ; 1$ to $25 \mathrm{~km} ; 25$ to 100 $\mathrm{km}$; more than $100 \mathrm{~km}$ ). Similarly, in a third model, we consider the frequency of contact between the parent and the (most frequently contacted) child. This variable distinguishes those with no children; interviewees with at least one child living in the same house(hold); parents having contact with the child on a daily basis; several times a week or weekly; less than weekly. In case of more than one child, proximity and contact refer to the child living closest and to the most frequently contacted child, respectively. We include as control socio-economic variables that were found to have an effect on cognitive functioning: marital status (married or living together with the partner; never married; separated or divorced; widowed), age, education (none or primary school: ISCED 0-1; lower secondary: ISCED 2; higher secondary and tertiary education: ISCED 3-4 and 56). Additionally, a three-category variable distinguishes retired persons from homemakers and controls for serious health conditions (i.e. "permanently sick or disabled"). Although physical health is increasingly visible in the theoretical and empirical models as an explanatory factor of cognitive decline, the conjoint trajectories of health conditions and cognition remain complex (Spiro and Brady 2008). To account for health-related contextual factors, we therefore include three variables indicating whether the respondent reports less than good health (good, very good $=0$; fair, bad, very bad $=1$ ), the depression level (based on the EURO$\mathrm{D}$ scale ranging between $1=$ not depressed and $12=$ depressed $^{2}$ and the frequency of engagement in vigorous physical activity, such as sports, heavy housework or a job that involves physical labour $(0=$ once a month or less; $1=$ once a week or more). Additionally, a measure of the maximum grip strength is included as a biomarker indicator: following SHARE instructions, two grip strength measurements on each hand were recorded with a dynamometer at the interview; the maximum of grip strength measures is defined as the maximum

1 All children alive are accounted for, with about $6 \%$ of the sample having one or more children not of their own, i.e. step child(ren), adopted, foster, or a child(ren) of the partner from a previous relationship.

2 The following 12 variables were used in SHARE to form the EURO-D scale: euro1: depression; euro2: pessimism; euro3: suicidality; euro4: guilt; euro5: sleep; euro6: interest; euro7: irritability; euro8: appetite; euro9: fatigue; euro10: concentration; eurol1: enjoyment; euro 12: tearfulness. 
grip strength measurement of both hands or of one hand, if grip strength was measured twice only on one hand. The handheld dynamometer used for this test measured grip strength from 0 to $100 \mathrm{~kg}$.

\section{Results}

\subsection{Descriptive findings}

The study sample counts slightly more women than men (56\% of the sample), with a mean age of 72.3 and 71.5, respectively (Table 1 and Table 2).

Reflecting the longer life expectancy of women, the sample shows more than twice as many widows than widowers and almost twice as many married men than women, which is typical among the non-institutionalised elderly population in ageing societies. As it could be expected for the cohorts considered in this study, men are on average more highly educated than women. The large majority of the sample declare themselves as retired. Confirming the lower female participation in the labour market, Mediterranean women show high proportions of homemakers, reaching almost $70 \%$ in the Spanish sample. About $2 \%$ of respondents declare themselves as permanently sick or disabled, while about half of the female sample $(50.7 \%)$ and $42.5 \%$ of the male sample report their health to be less than good. Concerning the other health-related contextual variables, descriptive findings show a mean depression level of 2.9 for women and 1.9 for men, on the EURO-D scale between 1 and 12, where 12 is depressed. Moreover, about $35 \%$ of women and $44 \%$ of men report to engage in vigorous physical activity more than once a week. In terms of the biomarker included in this study, the maximum of grip strength measure was on average about 24 among women and 39 among men, with country-specific differences of 6 points within the female sample and of more than 10 points within the male sample.

In our data, $14 \%$ of women and about $13 \%$ of men are childless, while most of the interviewees have two children (32.4\% of women and $35.5 \%$ of men) and around $15 \%$ have four children or more. In terms of proximity and frequency of contact to the children, there is a large variation across the SHARE countries. In northern European countries, only small proportions of the sample live together (i.e. in the same house or the same building) with at least one child. By contrast, Mediterranean mothers and fathers report this living condition as the most common one. European parents seem to generally have frequent contact with their children. In none of the countries considered, the sample reporting low frequency of contact (i.e. less than once a week) is more than $7.5 \%$ among women or $12 \%$ among men. ${ }^{3}$

3 With the exception of Swiss men, who were almost $17 \%$ in this category. 
Table 1:

Distribution of variables (\%) for women

\begin{tabular}{|c|c|c|c|c|c|c|c|c|c|c|c|c|}
\hline & $\mathbf{A T}$ & DE & SE & NL & ES & IT & FR & DK & GR & $\mathbf{C H}$ & BE & $\mathbf{A L L}$ \\
\hline \multicolumn{13}{|l|}{ Number of children } \\
\hline 0 & 18.3 & 13.2 & 11.8 & 13.4 & 14.2 & 15.2 & 13.6 & 11.5 & 12.8 & 17.6 & 13.6 & 14.0 \\
\hline 1 & 25.1 & 25.9 & 18.4 & 9.7 & 15.7 & 18.7 & 22.5 & 15.1 & 18.1 & 23.5 & 20.0 & 19.4 \\
\hline 2 & 32.4 & 31.5 & 37.8 & 31.0 & 28.8 & 31.6 & 27.0 & 36.4 & 45.3 & 25.3 & 27.8 & 32.4 \\
\hline 3 & 13.7 & 18.0 & 20.1 & 22.5 & 19.6 & 20.3 & 20.2 & 21.0 & 16.4 & 18.2 & 19.4 & 19.1 \\
\hline $4+$ & 10.5 & 11.3 & 11.8 & 23.3 & 21.6 & 14.1 & 16.8 & 15.9 & 7.5 & 15.3 & 19.1 & 15.2 \\
\hline \multicolumn{13}{|l|}{ Proximity } \\
\hline \multicolumn{13}{|l|}{ Child(ren) in } \\
\hline$<1 \mathrm{~km}$ & 13.6 & 12.7 & 16.8 & 21.9 & 22.4 & 13.0 & 15.4 & 20.7 & 16.1 & 14.7 & 24.1 & 17.4 \\
\hline $1-25 \mathrm{~km}$ & 29.8 & 34.2 & 43.8 & 45.7 & 15.7 & 19.7 & 38.3 & 46.8 & 22.2 & 30.6 & 41.7 & 33.0 \\
\hline $25-100 \mathrm{~km}$ & 6.7 & 7.6 & 13.8 & 9.1 & 2.3 & 3.6 & 8.9 & 10.9 & 3.2 & 10.6 & 5.2 & 7.0 \\
\hline$>100 \mathrm{~km}$ & 5.2 & 7.9 & 11.3 & 3.0 & 4.1 & 2.1 & 10.7 & 6.4 & 5.6 & 7.6 & 1.7 & 5.8 \\
\hline \multicolumn{13}{|l|}{ Contact } \\
\hline Daily & 19.3 & 18.2 & 28.3 & 28.3 & 28.6 & 26.1 & 24.9 & 28.9 & 28.9 & 18.8 & 31.0 & 25.9 \\
\hline Several times a week & & & & & & & & & & & & \\
\hline $\begin{array}{l}\text { or once a week } \\
\text { Less than weekly }\end{array}$ & $\begin{array}{r}29.3 \\
6.7\end{array}$ & $\begin{array}{r}37.1 \\
7.1\end{array}$ & $\begin{array}{r}53.2 \\
4.2\end{array}$ & $\begin{array}{r}47.6 \\
3.8\end{array}$ & $\begin{array}{r}14.7 \\
1.1\end{array}$ & $\begin{array}{r}11.3 \\
1.1\end{array}$ & $\begin{array}{r}40.9 \\
7.5\end{array}$ & $\begin{array}{r}50.7 \\
5.3\end{array}$ & $\begin{array}{r}17.0 \\
1.3\end{array}$ & $\begin{array}{r}38.2 \\
6.5\end{array}$ & $\begin{array}{r}36.7 \\
5.1\end{array}$ & $\begin{array}{l}33.0 \\
4.4\end{array}$ \\
\hline \multicolumn{13}{|l|}{ Marital status } \\
\hline Living with partner & 22.2 & 49.7 & 50.4 & 50.0 & 51.1 & 53.3 & 31.2 & 30.0 & 29.1 & 25.9 & 32.9 & 40.0 \\
\hline Never $\mathrm{n}$ & 11.4 & 5.4 & 5.5 & 6.7 & 8.0 & 8.3 & 7.5 & 4.8 & 5.2 & 8.2 & 5.9 & 7.0 \\
\hline Separated / divorced & 13.2 & 7.9 & 11.3 & 9.1 & 1.8 & 1.8 & 10.6 & 15.7 & 5.8 & 11.2 & 10.0 & 8.4 \\
\hline Widowed & 53.2 & 37.0 & 32.9 & 34.2 & 39.1 & 36.6 & 50.7 & 49.6 & 59.9 & 54.7 & 51.2 & 44.6 \\
\hline \multicolumn{13}{|l|}{ Age } \\
\hline Mean age & 71.7 & 71.3 & 73.5 & 71.4 & 72.9 & 70.3 & 73.5 & 73.8 & 72.1 & 74.2 & 72.7 & 72.3 \\
\hline \multicolumn{13}{|c|}{ 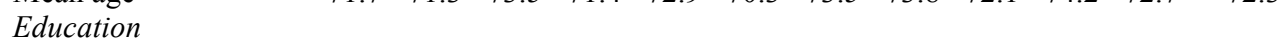 } \\
\hline None / primary & 31.1 & 1.4 & 51.1 & 27.5 & 82.2 & 76.2 & 62.3 & 36.4 & 76.0 & 32.4 & 40.9 & 48.8 \\
\hline Lower secondary & 16.5 & 34.9 & 14.8 & 46.6 & 12.4 & 10.7 & 9.2 & 12.3 & 8.5 & 40.0 & 22.0 & 19.3 \\
\hline $\begin{array}{l}\text { Higher secondary or } \\
\text { tertiary }\end{array}$ & 52.4 & 63.7 & 34.1 & 25.9 & 5.4 & 13.1 & 28.5 & 51.3 & 15.5 & 27.6 & 37.1 & 31.9 \\
\hline \multicolumn{13}{|c|}{ Working status and health } \\
\hline Retired & 82.1 & 81.2 & 97.5 & 43.9 & 27.0 & 63.3 & 80.4 & 94.4 & 54.7 & 92.9 & 71.3 & 69.6 \\
\hline Homemaker & 17.7 & 17.7 & 1.8 & 48.2 & 69.4 & 35.5 & 19.0 & 2.2 & 43.3 & 5.9 & 27.5 & 28.4 \\
\hline $\begin{array}{l}\text { Permanently sick or } \\
\text { disabled }\end{array}$ & 0.2 & 1.1 & 0.7 & 7.9 & 3.6 & 1.2 & 0.6 & 3.4 & 2.0 & 1.2 & 1.2 & 2.0 \\
\hline Less than good health & 49.5 & 57.0 & 45.4 & 36.6 & 65.3 & 63.4 & 48.9 & 41.2 & 55.8 & 27.1 & 43.3 & 50.7 \\
\hline Mean depression & 2.5 & 2.6 & 2.5 & 2.5 & 4.2 & 3.5 & 3.3 & 2.1 & 2.9 & 2.3 & 2.9 & 2.9 \\
\hline Vigorous activitie & & & & & & & & & & & & \\
\hline$\geq 1$ week & 31.5 & 9.1 & 38.9 & 46.6 & 25.9 & 30.0 & 28.8 & 41.7 & 42.1 & 43.5 & 29.0 & 34.9 \\
\hline \multicolumn{13}{|l|}{ Biomarker } \\
\hline Mean grip strength & 25.9 & 26.3 & 24.0 & 25.0 & 20.3 & 22.1 & 23.0 & 23.9 & 22.9 & 23.4 & 23.8 & 23.7 \\
\hline $\mathrm{N}$ & 553 & 644 & 566 & 506 & 611 & 664 & 663 & 357 & 554 & 170 & 690 & 5978 \\
\hline
\end{tabular}

Source: SHARE Release 2.5.0, authors' calculations. 
Table 2:

Distribution of variables (\%) for men

\begin{tabular}{|c|c|c|c|c|c|c|c|c|c|c|c|c|}
\hline & AT & DE & SE & NL & ES & IT & FR & DK & GR & $\mathbf{C H}$ & BE & $\overline{\mathbf{A L L}}$ \\
\hline \multicolumn{13}{|l|}{ Number of children } \\
\hline 0 & 14.5 & 16.3 & 9.6 & 7.1 & 11.3 & 14.4 & 15.8 & 12.5 & 11.1 & 15.1 & 12.9 & 12.7 \\
\hline 1 & 21.9 & 20.2 & 11.5 & 12.6 & 11.1 & 20.3 & 17.8 & 11.3 & 18.2 & 17.3 & 21.5 & 17.1 \\
\hline 2 & 35.7 & 35.0 & 38.2 & 40.4 & 29.3 & 36.2 & 27.5 & 40.7 & 48.3 & 31.8 & 31.5 & 35.5 \\
\hline 3 & 17.5 & 18.9 & 21.9 & 20.6 & 23.3 & 16.2 & 19.6 & 19.8 & 15.6 & 21.2 & 18.0 & 19.2 \\
\hline $4+$ & 10.4 & 9.5 & 18.7 & 19.3 & 25 & 13 & 19.4 & 15.7 & 6.7 & 14.5 & 16.2 & 15.4 \\
\hline \multicolumn{13}{|l|}{ Proximity } \\
\hline \multicolumn{13}{|l|}{ Child(ren) in } \\
\hline house(hold) & 24.2 & 18.1 & 3.8 & 7.8 & 36.3 & 44.8 & 12.6 & 4.8 & 39.6 & 15.1 & 12.4 & 20.1 \\
\hline$<1 \mathrm{~km}$ & 13.5 & 12.2 & 16.3 & 24.2 & 26.8 & 14.2 & 13.4 & 12.5 & 19.2 & 10.6 & 21.5 & 17.5 \\
\hline $1-25 \mathrm{~km}$ & 31.2 & 34.4 & 45.3 & 49.9 & 18.9 & 19.4 & 33.9 & 46.8 & 20.6 & 41.9 & 43.5 & 34.9 \\
\hline $25-100 \mathrm{~km}$ & 7.5 & 9.4 & 12.5 & 7.3 & 2.1 & 3.3 & 12.2 & 15.7 & 2.6 & 9.5 & 6.9 & 7.7 \\
\hline$>100 \mathrm{~km}$ & 9.2 & 9.6 & 12.5 & 3.8 & 4.6 & 3.9 & 12.2 & 7.7 & 6.9 & 7.8 & 2.8 & 7.2 \\
\hline \multicolumn{13}{|l|}{ Contact } \\
\hline Daily & 15.2 & 16.5 & 29.6 & 29.7 & 33.3 & 25.5 & 23.0 & 19.8 & 31.3 & 9.5 & 29.1 & 25.1 \\
\hline \multicolumn{13}{|l|}{ Several times a week } \\
\hline or once a week & 34.4 & 39.7 & 50.3 & 49.0 & 15.7 & 12.4 & 39.1 & 52.4 & 17.1 & 43.6 & 39.2 & 35.1 \\
\hline Less than weekly & 11.7 & 9.4 & 6.7 & 6.4 & 3.5 & 2.8 & 9.6 & 10.5 & 0.9 & 16.8 & 6.4 & 7.0 \\
\hline \multicolumn{13}{|l|}{ Marital status } \\
\hline Living with partner & 70.8 & 77.8 & 74.3 & 78.5 & 79.4 & 79.9 & 70.3 & 57.3 & 74.6 & 73.2 & 75.2 & 74.6 \\
\hline $\mathrm{Nev}$ & 7.2 & 7.5 & 5.2 & 3.8 & 7.2 & 9.0 & 8.0 & 6.0 & 3.3 & 5.0 & 4.2 & 6.0 \\
\hline / divorced & 8.0 & 4.9 & 8.8 & 4.2 & 1.4 & 2.0 & 9.0 & 15.7 & 5.2 & 8.4 & 5.5 & 6.1 \\
\hline Wido & 14.0 & 9.8 & 11.7 & 13.5 & 12.0 & 9.2 & 12.8 & 21.0 & 16.8 & 13.4 & 15.1 & 13.3 \\
\hline \multicolumn{13}{|l|}{ Age } \\
\hline Mean age & 9.4 & 69.6 & 2.9 & 71.2 & 72.7 & 70.1 & 71.8 & 72.2 & 72.2 & 72.8 & 72.1 & 71.5 \\
\hline \multicolumn{13}{|l|}{ Education } \\
\hline None / primar & 16.7 & 0.4 & 52.4 & 21.5 & 74.4 & 61.4 & 51.3 & 21.8 & 57.6 & 19.6 & 31.7 & 38.4 \\
\hline Lower secondary & 6.0 & 7.3 & 15.2 & 36.8 & 13.4 & 20.5 & 5.8 & 2.8 & 10.2 & 25.7 & 20.8 & 15.2 \\
\hline $\begin{array}{l}\text { Higher secondary or } \\
\text { tertiary }\end{array}$ & Higher secondary or & 92.3 & 32.4 & 41.7 & 12.2 & 18.1 & 42.9 & 75.4 & 32.2 & 54.7 & 47.4 & 46.4 \\
\hline \multicolumn{13}{|c|}{ Working status and health } \\
\hline Retired & 98.3 & 96.7 & 97.7 & 92.7 & 96.8 & 98.7 & 98.8 & 97.6 & 99.1 & 95.0 & 96.5 & 97.1 \\
\hline Homemaker & 0.5 & 0.2 & 0.2 & 1.6 & 0.9 & 0.2 & 0.4 & 0.0 & 0.0 & 0.6 & 0.6 & 0.5 \\
\hline $\begin{array}{l}\text { Permanently sick or } \\
\text { disabled }\end{array}$ & 1.2 & 3.1 & 2.1 & 5.8 & 2.3 & 1.1 & 0.8 & 2.4 & 0.9 & 4.5 & 2.9 & 2.4 \\
\hline Less than good health & 38.4 & 52.8 & 38.8 & 37.3 & 52 & 54.4 & 47.: & 38.3 & 42.7 & $21 . \varepsilon$ & 33.1 & 42. \\
\hline Mean depression & 1.4 & 1.7 & 1.7 & 1.7 & 2.5 & 2.5 & 2.4 & 1.6 & 1.9 & 1.7 & 1.8 & 1.9 \\
\hline \multicolumn{13}{|l|}{ Vigorous activities } \\
\hline$\geq 1$ week & 47.4 & 3.6 & 53.0 & 41.7 & 29.3 & 41.7 & 36.7 & 52.8 & 41.2 & 58.1 & 39.2 & 43.7 \\
\hline \multicolumn{13}{|l|}{ Biomarker } \\
\hline Mean grip strength & 43.6 & 42.5 & 40.4 & 41.5 & 33.1 & 36.8 & 38.0 & 40.8 & 36.8 & 39.6 & 39.8 & 39.3 \\
\hline $\mathrm{N}$ & 401 & 491 & 479 & 451 & 433 & 458 & 501 & 248 & 422 & 179 & 715 & 4778 \\
\hline
\end{tabular}

Source: SHARE Release 2.5.0, authors' calculations.

Descriptive results indicate an average level of fluency of 17.9 for men and 16.5 for women (i.e., on average, men name about 18 animals within one minute and women almost 17). The scores for immediate recall are about 4.2 for both 
men and women (i.e. respondents recall on average about 4 words out of ten immediately after they have been read to them); while those for delayed recall are 2.7 and 2.8 , respectively (i.e. respondents recall slightly less than 3 words five minutes later). Figure 1 shows the distribution of the three cognitive tests across the eleven countries participating in the first wave of SHARE. The highest levels of fluency abilities were recorded in Sweden and Austria for both men and women. The lowest levels were found among Mediterranean samples. The highest levels of immediate recall were recorded in Germany and Switzerland for men and in Denmark and Germany for women. Concerning delayed recall, Switzerland registers the highest level among men, followed by Germany; while Denmark leaves the other countries behind among women. The lowest levels of both immediate and delayed recall are found in Spain.

Figure 1:

Average number of words (a) named during the fluency test; (b) recalled during the immediate recall test; (c) recalled during the delayed recall test, among men and women, by country

(a)

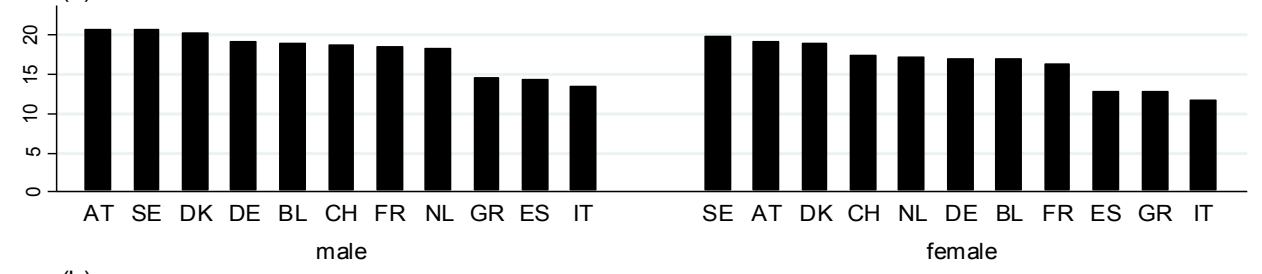

(b)

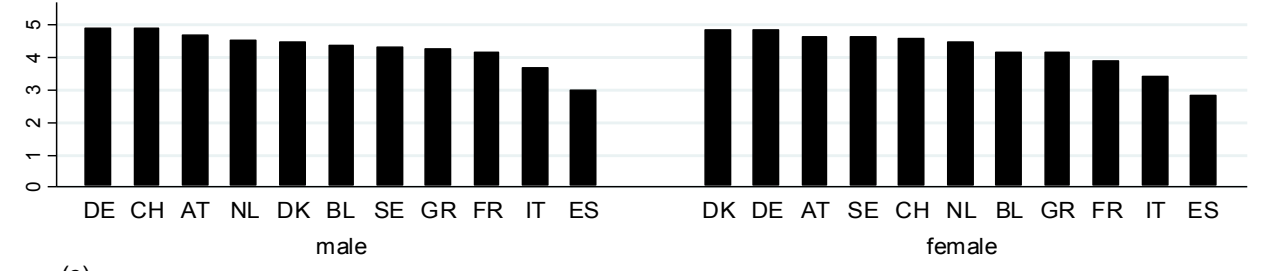

(c)

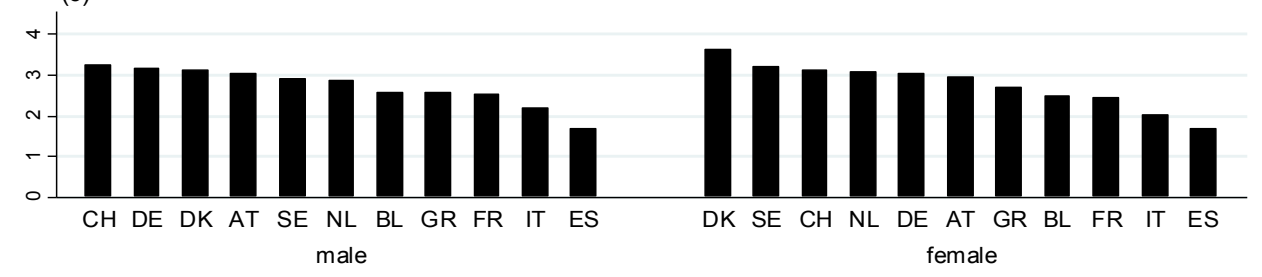

Note: Sorted in descending order according to the number of words named or recalled.

Source: SHARE Release 2.5.0, sample aged 60 and older and not in the labour force, weighted by calibrated weights, Wave 1 prepared for household main/dropoff sample. 


\subsection{Multivariate results}

Multivariate regression models are estimated separately for men and women, the dependent variable being first fluency (Table 3), second immediate recall (Table 4), third delayed recall (Table 5). In each table, Model 1 includes number of children as explanatory variable; the explanatory variable in Model 2 is local proximity to the closest child; while most frequent contact to the child(ren) is the explanatory variable of Model 3.

Table 3:

Estimated coefficients from regressions for fluency among older women and men

\begin{tabular}{|c|c|c|c|c|c|c|}
\hline a. Explanatory variables & Model 1 & Women & Model 3 & \multicolumn{3}{|c|}{ Men } \\
\hline \multicolumn{7}{|l|}{ Number of children (Ref. 2) } \\
\hline 0 & $\begin{array}{l}-0.591 * \\
(0.290)\end{array}$ & & & $\begin{array}{l}-0.816^{*} \\
(0.350)\end{array}$ & & \\
\hline 1 child & $\begin{array}{l}-0.295 \\
(0.221)\end{array}$ & & & $\begin{array}{l}-0.819^{* *} \\
(0.258)\end{array}$ & & \\
\hline 3 & $\begin{array}{l}-0.357+ \\
(0.220)\end{array}$ & & & $\begin{array}{l}-0.155 \\
(0.248)\end{array}$ & & \\
\hline $4+$ & $\begin{array}{c}0.097 \\
(0.240)\end{array}$ & & & $\begin{array}{l}-0.627 * \\
(0.270)\end{array}$ & & \\
\hline \multicolumn{7}{|c|}{ Location of child ${ }^{l}$ (Ref. 0) } \\
\hline Same house(hold) & & $\begin{array}{c}0.029 \\
(0.306)\end{array}$ & & & $\begin{array}{c}0.054 \\
(0.381)\end{array}$ & \\
\hline$<1 \mathrm{~km}$ & & $\begin{array}{c}0.320 \\
(0.315)\end{array}$ & & & $\begin{array}{c}0.054 \\
(0.382)\end{array}$ & \\
\hline $1-25 \mathrm{~km}$ & & $\begin{array}{c}0.626^{*} \\
(0.289)\end{array}$ & & & $\begin{array}{c}0.704^{*} \\
(0.351)\end{array}$ & \\
\hline $25-100 \mathrm{~km}$ & & $\begin{array}{l}1.215^{* *} \\
(0.384)\end{array}$ & & & $\begin{array}{r}0.979^{*} \\
(0.447)\end{array}$ & \\
\hline$>100 \mathrm{~km}$ & & $\begin{array}{c}0.516 \\
(0.405)\end{array}$ & & & $\begin{array}{r}0.977 * \\
(0.455)\end{array}$ & \\
\hline \multicolumn{7}{|c|}{ Contact with child(ren) ${ }^{2}$ (Ref. 0) } \\
\hline \multicolumn{2}{|c|}{ Child(ren) in house(hold) } & & $\begin{array}{c}0.043 \\
(0.306)\end{array}$ & & & $\begin{array}{c}0.084 \\
(0.381)\end{array}$ \\
\hline Daily & & & $\begin{array}{c}0.448 \\
(0.297)\end{array}$ & & & $\begin{array}{c}0.420 \\
(0.365)\end{array}$ \\
\hline \multicolumn{2}{|l|}{ Once or several times a week } & & $\begin{array}{l}0.805^{* *} \\
(0.290)\end{array}$ & & & $\begin{array}{l}0.857 * * \\
(0.351)\end{array}$ \\
\hline Less than weekly & & & $\begin{array}{c}0.119 \\
(0.442) \\
\end{array}$ & & & $\begin{array}{c}0.119 \\
(0.457) \\
\end{array}$ \\
\hline \multicolumn{7}{|l|}{ b. Control variables } \\
\hline \multicolumn{7}{|c|}{ Marital status (Ref. living with partner) } \\
\hline Never married & $\begin{array}{l}-0.164 \\
(0.383)\end{array}$ & $\begin{array}{l}-0.166 \\
(0.382)\end{array}$ & $\begin{array}{l}-0.137 \\
(0.383)\end{array}$ & $\begin{array}{l}-1.271^{* *} \\
(0.468)\end{array}$ & $\begin{array}{l}-1.351 * * \\
(0.468)\end{array}$ & $\begin{array}{l}-1.304 * * \\
(0.469)\end{array}$ \\
\hline Separated / divorced & $\begin{array}{l}-0.139 \\
(0.296)\end{array}$ & $\begin{array}{l}-0.173 \\
(0.296)\end{array}$ & $\begin{array}{l}-0.132 \\
(0.297)\end{array}$ & $\begin{array}{c}0.115 \\
(0.373)\end{array}$ & $\begin{array}{l}-0.052 \\
(0.374)\end{array}$ & $\begin{array}{c}0.083 \\
(0.380)\end{array}$ \\
\hline Widowed & $\begin{array}{l}-0.309+ \\
(0.187) \\
\end{array}$ & $\begin{array}{l}-0.268 \\
(0.188) \\
\end{array}$ & $\begin{array}{l}-0.255 \\
(0.188) \\
\end{array}$ & $\begin{array}{c}0.232 \\
(0.276) \\
\end{array}$ & $\begin{array}{c}0.192 \\
(0.276) \\
\end{array}$ & $\begin{array}{c}0.240 \\
(0.277) \\
\end{array}$ \\
\hline
\end{tabular}




\begin{tabular}{|c|c|c|c|c|c|c|}
\hline b. Control variables & Model 1 & Women & Model 3 & \multicolumn{3}{|c|}{ Men } \\
\hline \multicolumn{7}{|l|}{ Age } \\
\hline Age & $\begin{array}{l}-0.046 \\
(0.149)\end{array}$ & $\begin{array}{l}-0.073 \\
(0.149)\end{array}$ & $\begin{array}{l}-0.078 \\
(0.150)\end{array}$ & $\begin{array}{r}0.374^{*} \\
(0.185)\end{array}$ & $\begin{array}{c}0.354+ \\
(0.185)\end{array}$ & $\begin{array}{c}0.339+ \\
(0.185)\end{array}$ \\
\hline Age*age $/ 100$ & $\begin{array}{l}-0.072 \\
(0.100)\end{array}$ & $\begin{array}{l}-0.055 \\
(0.100)\end{array}$ & $\begin{array}{l}-0.052 \\
(0.100)\end{array}$ & $\begin{array}{l}-0.331 * * \\
(0.125)\end{array}$ & $\begin{array}{l}-0.319^{*} \\
(0.125)\end{array}$ & $\begin{array}{l}-0.310^{*} \\
(0.125)\end{array}$ \\
\hline \multicolumn{7}{|c|}{ Education (Ref. none / primary) } \\
\hline Lower secondary & $\begin{array}{l}1.344 * * * \\
(0.226)\end{array}$ & $\begin{array}{l}1.309^{* * *} \\
(0.226)\end{array}$ & $\begin{array}{l}1.326^{* * *} \\
(0.226)\end{array}$ & $\begin{array}{l}1.812 * * * \\
(0.277)\end{array}$ & $\begin{array}{l}1.799 * * * \\
(0.277)\end{array}$ & $\begin{array}{l}1.823 * * * \\
(0.277)\end{array}$ \\
\hline $\begin{array}{l}\text { Higher secondary or } \\
\text { tertiary }\end{array}$ & $\begin{array}{l}3.357 * * * \\
(0.205)\end{array}$ & $\begin{array}{l}3.276^{* * *} \\
(0.206)\end{array}$ & $\begin{array}{l}3.296^{* * *} \\
(0.205)\end{array}$ & $\begin{array}{l}3.566 * * * \\
(0.223)\end{array}$ & $\begin{array}{l}3.511^{* * *} \\
(0.224)\end{array}$ & $\begin{array}{l}3.573 * * * \\
(0.223)\end{array}$ \\
\hline \multicolumn{7}{|c|}{ Working status and health } \\
\hline $\begin{array}{l}\text { Homemaker (Ref. } \\
\text { retired) }\end{array}$ & $\begin{array}{l}-1.164 * \\
(0.567)\end{array}$ & $\begin{array}{l}-1.201^{*} \\
(0.566)\end{array}$ & $\begin{array}{l}-1.158^{*} \\
(0.566)\end{array}$ & $\begin{array}{l}-0.660 \\
(0.600)\end{array}$ & $\begin{array}{l}-0.584 \\
(0.600)\end{array}$ & $\begin{array}{l}-0.589 \\
(0.600)\end{array}$ \\
\hline $\begin{array}{l}\text { Permanently sick or } \\
\text { disabled }\end{array}$ & $\begin{array}{l}-0.248 \\
(0.194)\end{array}$ & $\begin{array}{l}-0.207 \\
(0.193)\end{array}$ & $\begin{array}{l}-0.222 \\
(0.193)\end{array}$ & $\begin{array}{l}0.610 \\
(1.264)\end{array}$ & $\begin{array}{c}0.722 \\
(1.265)\end{array}$ & $\begin{array}{l}0.587 \\
(1.265)\end{array}$ \\
\hline Less than good health & $-0.935^{* * *}$ & $-0.922 * * *$ & $-0.919 * * *$ & $-1.117 * * *$ & $-1.119^{* * *}$ & $-1.096^{* * *}$ \\
\hline $\begin{array}{l}\text { (Ref. good/very good) } \\
\text { Euro-D depression scale }\end{array}$ & $\begin{array}{l}(0.173) \\
-0.108 * *\end{array}$ & $\begin{array}{l}(0.173) \\
-0.110 * *\end{array}$ & $\begin{array}{l}(0.173) \\
-0.108 * *\end{array}$ & $\begin{array}{l}(0.198) \\
-0.224 * * *\end{array}$ & $\begin{array}{l}(0.198) \\
-0.224 * * *\end{array}$ & $\begin{array}{l}(0.198) \\
-0.223 * * *\end{array}$ \\
\hline (Ref. not depr.) & $(0.035)$ & $(0.035)$ & $(0.035)$ & $(0.047)$ & $(0.047)$ & $(0.047)$ \\
\hline $\begin{array}{l}\text { Vigorous activities } \geq 1 \text { a } \\
\text { week (Ref. less) }\end{array}$ & $\begin{array}{l}1.085^{* * *} \\
(0.172)\end{array}$ & $\begin{array}{l}1.072^{* * *} \\
(0.172)\end{array}$ & $\begin{array}{l}1.071 * * * \\
(0.172)\end{array}$ & $\begin{array}{l}0.612 * * \\
(0.189)\end{array}$ & $\begin{array}{l}0.613^{* *} \\
(0.189)\end{array}$ & $\begin{array}{l}0.621 * * \\
(0.189)\end{array}$ \\
\hline \multicolumn{7}{|l|}{ Biomarker } \\
\hline Grip strength (Ref. 1) & $\begin{array}{l}0.114 * * * \\
(0.013)\end{array}$ & $\begin{array}{l}0.113^{* * *} \\
(0.013)\end{array}$ & $\begin{array}{l}0.114 * * * \\
(0.013)\end{array}$ & $\begin{array}{l}0.080^{* * *} \\
(0.011)\end{array}$ & $\begin{array}{l}0.080^{* * *} \\
(0.011)\end{array}$ & $\begin{array}{l}0.080^{* * *} \\
(0.011)\end{array}$ \\
\hline \multicolumn{7}{|l|}{ Country (Ref. Austria) } \\
\hline Germany & $\begin{array}{l}-2.910 * * * \\
(0.349)\end{array}$ & $\begin{array}{l}-2.924 * * * \\
(0.349)\end{array}$ & $\begin{array}{l}-2.933^{* * *} \\
(0.349)\end{array}$ & $\begin{array}{l}-1.781^{* * *} \\
(0.409)\end{array}$ & $\begin{array}{l}-1.814 * * * \\
(0.409)\end{array}$ & $\begin{array}{l}-1.824 * * * \\
(0.409)\end{array}$ \\
\hline Sweden & $\begin{array}{l}1.306^{* * *} \\
(0.361)\end{array}$ & $\begin{array}{l}1.139^{* *} \\
(0.365)\end{array}$ & $\begin{array}{l}1.136^{* *} \\
(0.365)\end{array}$ & $\begin{array}{l}2.053 * * * \\
(0.421)\end{array}$ & $\begin{array}{l}1.937 * * * \\
(0.424)\end{array}$ & $\begin{array}{l}1.964 * * * \\
(0.424)\end{array}$ \\
\hline Netherlands & $\begin{array}{l}-1.779 * * * \\
(0.381)\end{array}$ & $\begin{array}{l}-1.873 * * * \\
(0.382)\end{array}$ & $\begin{array}{l}-1.903 * * * \\
(0.382)\end{array}$ & $\begin{array}{l}-1.562 * * * \\
(0.426)\end{array}$ & $\begin{array}{l}-1.580 * * * \\
(0.428)\end{array}$ & $\begin{array}{l}-1.647^{* * *} \\
(0.428)\end{array}$ \\
\hline Spain & $\begin{array}{l}-4.559^{* * *} \\
(0.381)\end{array}$ & $\begin{array}{l}-4.462 * * * \\
(0.381)\end{array}$ & $\begin{array}{l}-4.479 * * * \\
(0.382)\end{array}$ & $\begin{array}{l}-3.051^{* * *} \\
(0.451)\end{array}$ & $\begin{array}{l}-2.893^{* * * *} \\
(0.451)\end{array}$ & $\begin{array}{l}-2.934 * * * \\
(0.452)\end{array}$ \\
\hline Italy & $\begin{array}{l}-6.359 * * * \\
(0.359)\end{array}$ & $\begin{array}{l}-6.274 * * * \\
(0.360)\end{array}$ & $\begin{array}{l}-6.256^{* * *} \\
(0.361)\end{array}$ & $\begin{array}{l}-4.244 * * * \\
(0.436)\end{array}$ & $\begin{array}{l}-4.111^{* * * *} \\
(0.438)\end{array}$ & $\begin{array}{l}-4.101^{* * *} \\
(0.440)\end{array}$ \\
\hline France & $\begin{array}{l}-1.443^{* * *} \\
(0.346)\end{array}$ & $\begin{array}{l}-1.553 * * * \\
(0.348)\end{array}$ & $\begin{array}{l}-1.547 * * * \\
(0.347)\end{array}$ & $\begin{array}{l}-0.292 \\
(0.414)\end{array}$ & $\begin{array}{l}-0.419 \\
(0.415)\end{array}$ & $\begin{array}{l}-0.374 \\
(0.415)\end{array}$ \\
\hline Denmark & $\begin{array}{l}-0.723+ \\
(0.404)\end{array}$ & $\begin{array}{l}-0.842^{*} \\
(0.406)\end{array}$ & $\begin{array}{l}-0.865^{*} \\
(0.406)\end{array}$ & $\begin{array}{l}-0.397 \\
(0.490)\end{array}$ & $\begin{array}{l}-0.470 \\
(0.491)\end{array}$ & $\begin{array}{l}-0.472 \\
(0.491)\end{array}$ \\
\hline Greece & $\begin{array}{l}-5.607 * * * \\
(0.369)\end{array}$ & $\begin{array}{l}-5.539 * * * \\
(0.368)\end{array}$ & $\begin{array}{l}-5.542 * * * \\
(0.369)\end{array}$ & $\begin{array}{l}-4.239 * * * \\
(0.435)\end{array}$ & $\begin{array}{l}-4.036 * * * \\
(0.436)\end{array}$ & $\begin{array}{l}-4.059 * * * \\
(0.439)\end{array}$ \\
\hline Switzerland & $\begin{array}{l}-1.587 * * \\
(0.522)\end{array}$ & $\begin{array}{l}-1.655^{* *} \\
(0.522)\end{array}$ & $\begin{array}{l}-1.652 * * \\
(0.522)\end{array}$ & $\begin{array}{l}-1.436 * * \\
(0.549)\end{array}$ & $\begin{array}{l}-1.509^{* *} \\
(0.550)\end{array}$ & $\begin{array}{l}-1.471^{* *} \\
(0.550)\end{array}$ \\
\hline Belgium & $\begin{array}{l}-1.749 * * * \\
(0.340)\end{array}$ & $\begin{array}{l}-1.798^{* * *} \\
(0.341)\end{array}$ & $\begin{array}{l}-1.827 * * * \\
(0.340)\end{array}$ & $\begin{array}{c}-0.848^{*} \\
(0.384)\end{array}$ & $\begin{array}{l}-0.893 * \\
(0.385)\end{array}$ & $\begin{array}{c}-0.937^{*} \\
(0.385)\end{array}$ \\
\hline cons & $\begin{array}{l}22.907 * * * \\
(5.553)\end{array}$ & $\begin{array}{l}23.476^{* * * *} \\
(5.565)\end{array}$ & $\begin{array}{l}23.628^{* * * *} \\
(5.568)\end{array}$ & $\begin{array}{c}5.579 \\
(6.847)\end{array}$ & $\begin{array}{c}5.675 \\
(6.888)\end{array}$ & $\begin{array}{c}6.202 \\
(6.889)\end{array}$ \\
\hline $\mathrm{N}$ & 5978 & 5978 & 5978 & 4778 & 4778 & 4778 \\
\hline
\end{tabular}

Note: Standard errors in parentheses. $+\mathrm{p}<0.10,{ }^{*} \mathrm{p}<0.05,{ }^{*} \mathrm{p}<0.01,{ }^{* * *} \mathrm{p}<0.001$.

Source: SHARE Release 2.5.0, authors' calculations. 
Table 4:

Estimated coefficients from regressions for immediate recall among older women and men

\begin{tabular}{|c|c|c|c|c|c|c|}
\hline \multirow[b]{2}{*}{ a. Explanatory variables } & \multicolumn{3}{|c|}{ Women } & \multicolumn{3}{|c|}{ Men } \\
\hline & Model 1 & Model 2 & Model 3 & Model 1 & Model 2 & Model 3 \\
\hline \multicolumn{7}{|c|}{ Number of children (Ref. 2) } \\
\hline \multirow[t]{2}{*}{0} & -0.041 & & & 0.010 & & \\
\hline & $(0.075)$ & & & $(0.085)$ & & \\
\hline \multirow[t]{2}{*}{1 child } & $0.096+$ & & & -0.071 & & \\
\hline & $(0.057)$ & & & $(0.063)$ & & \\
\hline \multirow[t]{2}{*}{3} & $-0.114 *$ & & & 0.054 & & \\
\hline & $(0.057)$ & & & $(0.060)$ & & \\
\hline \multirow[t]{2}{*}{$4+$} & -0.040 & & & $-0.189 * *$ & & \\
\hline & $(0.062)$ & & & $(0.066)$ & & \\
\hline \multicolumn{7}{|c|}{ Location of child ${ }^{l}$ (Ref. 0) } \\
\hline \multirow[t]{2}{*}{ Same house(hold) } & & -0.097 & & & $-0.155^{+}$ & \\
\hline & & $(0.079)$ & & & $(0.093)$ & \\
\hline \multirow[t]{2}{*}{$<1 \mathrm{~km}$} & & 0.030 & & & -0.138 & \\
\hline & & $(0.081)$ & & & $(0.093)$ & \\
\hline \multirow[t]{2}{*}{$1-25 \mathrm{~km}$} & & 0.085 & & & 0.023 & \\
\hline & & $(0.075)$ & & & $(0.085)$ & \\
\hline \multirow[t]{2}{*}{$25-100 \mathrm{~km}$} & & $0.162^{+}$ & & & -0.051 & \\
\hline & & $(0.099)$ & & & $(0.109)$ & \\
\hline \multirow[t]{2}{*}{$>100 \mathrm{~km}$} & & 0.149 & & & 0.090 & \\
\hline & & $(0.105)$ & & & $(0.111)$ & \\
\hline \multicolumn{7}{|c|}{ Contact with child(ren) (Ref. 0) } \\
\hline \multirow[t]{2}{*}{ Child(ren) in house(hold) } & & & -0.088 & & & $-0.155^{+}$ \\
\hline & & & $(0.079)$ & & & $(0.093)$ \\
\hline \multirow[t]{2}{*}{ Daily } & & & 0.106 & & & -0.105 \\
\hline & & & $(0.077)$ & & & $(0.089)$ \\
\hline \multirow{2}{*}{\multicolumn{2}{|c|}{ Once or several times a week }} & & 0.095 & & & 0.072 \\
\hline & & & $(0.075)$ & & & $(0.086)$ \\
\hline \multirow[t]{2}{*}{ Less than weekly } & & & -0.071 & & & -0.136 \\
\hline & & & $(0.114)$ & & & $(0.111)$ \\
\hline \multicolumn{7}{|l|}{ b. Control variables } \\
\hline \multicolumn{7}{|c|}{ Marital status (Ref. living with partner) } \\
\hline \multirow[t]{2}{*}{ Never married } & $-0.181^{+}$ & $-0.166^{+}$ & -0.156 & $-0.189^{+}$ & $-0.203^{+}$ & $-0.195^{+}$ \\
\hline & $(0.099)$ & $(0.099)$ & $(0.099)$ & $(0.114)$ & $(0.114)$ & $(0.114)$ \\
\hline Separated / divorced & $-0.155^{*}$ & $-0.150 *$ & $-0.136^{+}$ & -0.083 & -0.116 & -0.091 \\
\hline & $(0.076)$ & $(0.076)$ & $(0.077)$ & $(0.091)$ & $(0.091)$ & $(0.093)$ \\
\hline Widowed & $-0.162 * * *$ & $-0.147 * *$ & $-0.145^{* *}$ & -0.035 & -0.038 & -0.029 \\
\hline & $(0.048)$ & $(0.048)$ & $(0.048)$ & $(0.067)$ & $(0.067)$ & $(0.067)$ \\
\hline Age & & & & & & \\
\hline Age & 0.012 & 0.005 & 0.005 & 0.043 & 0.039 & 0.034 \\
\hline & $(0.039)$ & $(0.039)$ & $(0.039)$ & $(0.045)$ & $(0.045)$ & $(0.045)$ \\
\hline Age*age/100 & $-0.046^{+}$ & -0.041 & -0.041 & $-0.062 *$ & $-0.060^{+}$ & $-0.057^{+}$ \\
\hline & $(0.026)$ & $(0.026)$ & $(0.026)$ & $(0.030)$ & $(0.031)$ & $(0.031)$ \\
\hline Education (Ref. none/pr & imary) & & & & & \\
\hline Lower secondary & $0.416^{* * *}$ & $0.415 * * *$ & $0.420 * * *$ & $0.639 * * *$ & $0.641 * * *$ & $0.646^{* * *}$ \\
\hline & $(0.058)$ & $(0.058)$ & $(0.058)$ & $(0.068)$ & $(0.068)$ & $(0.068)$ \\
\hline
\end{tabular}

(Table 4 continues on next page) 


\begin{tabular}{|c|c|c|c|c|c|c|}
\hline b. Control variables & \multicolumn{3}{|c|}{ Women } & Model 1 & $\begin{array}{c}\text { Men } \\
\text { Model } 2 \\
\end{array}$ & Model 3 \\
\hline $\begin{array}{l}\text { Higher secondary or } \\
\text { tertiary }\end{array}$ & $\begin{array}{l}0.943 * * * \\
(0.053)\end{array}$ & $\begin{array}{l}0.934 * * * \\
(0.053)\end{array}$ & $\begin{array}{l}0.944 * * * \\
(0.053)\end{array}$ & $\begin{array}{l}1.100^{* * *} \\
(0.054)\end{array}$ & $\begin{array}{l}1.094 * * * \\
(0.055)\end{array}$ & $\begin{array}{l}1.104 * * * \\
(0.054)\end{array}$ \\
\hline Working status and health & & & & & & \\
\hline Homemaker (Ref. retired) & $\begin{array}{l}-0.202 \\
(0.146)\end{array}$ & $\begin{array}{l}-0.213 \\
(0.146)\end{array}$ & $\begin{array}{l}-0.211 \\
(0.146)\end{array}$ & $\begin{array}{l}-0.206 \\
(0.146)\end{array}$ & $\begin{array}{l}-0.193 \\
(0.146)\end{array}$ & $\begin{array}{l}-0.193 \\
(0.146)\end{array}$ \\
\hline $\begin{array}{l}\text { Permanently sick or } \\
\text { disabled }\end{array}$ & $\begin{array}{l}-0.061 \\
(0.050)\end{array}$ & $\begin{array}{l}-0.062 \\
(0.050)\end{array}$ & $\begin{array}{l}-0.066 \\
(0.050)\end{array}$ & $\begin{array}{l}0.063 \\
(0.308)\end{array}$ & $\begin{array}{l}0.098 \\
(0.309)\end{array}$ & $\begin{array}{l}0.062 \\
(0.308)\end{array}$ \\
\hline Less than good health & $-0.234 * * *$ & $-0.226 * * *$ & $-0.226 * * *$ & $-0.176 * * *$ & $-0.179 * * *$ & $-0.170 * * *$ \\
\hline (Ref. good/very good) & $(0.045)$ & $(0.045)$ & $(0.045)$ & $(0.048)$ & $(0.048)$ & $(0.048)$ \\
\hline $\begin{array}{l}\text { Euro-D depression scale } \\
\text { (Ref. not depressed) }\end{array}$ & $\begin{array}{l}-0.065 * * * \\
(0.009)\end{array}$ & $\begin{array}{l}-0.066 * * * \\
(0.009)\end{array}$ & $\begin{array}{l}-0.065 * * * \\
(0.009)\end{array}$ & $\begin{array}{l}-0.085 * * * \\
(0.011)\end{array}$ & $\begin{array}{l}-0.085 * * * \\
(0.011)\end{array}$ & $\begin{array}{l}-0.085^{* * *} \\
(0.011)\end{array}$ \\
\hline $\begin{array}{l}\text { Vigorous activities } \geq 1 \text { a } \\
\text { week (Ref. less) }\end{array}$ & $\begin{array}{l}0.132^{* *} \\
(0.044)\end{array}$ & $\begin{array}{l}0.126^{* *} \\
(0.044)\end{array}$ & $\begin{array}{l}0.127^{* *} \\
(0.044)\end{array}$ & $\begin{array}{c}0.071 \\
(0.046)\end{array}$ & $\begin{array}{l}0.074 \\
(0.046)\end{array}$ & $\begin{array}{r}0.078^{+} \\
(0.046)\end{array}$ \\
\hline Biomarker & & & & & & \\
\hline Grip strength (Ref. 1) & $\begin{array}{l}0.021 * * * \\
(0.003)\end{array}$ & $\begin{array}{l}0.021 * * * \\
(0.003)\end{array}$ & $\begin{array}{l}0.021 * * * \\
(0.003)\end{array}$ & $\begin{array}{l}0.013 * * * \\
(0.003)\end{array}$ & $\begin{array}{l}0.013 * * * \\
(0.003)\end{array}$ & $\begin{array}{l}0.013 * * * \\
(0.003)\end{array}$ \\
\hline Country (Ref. Austria) & & & & & & \\
\hline Germany & $\begin{array}{c}0.032 \\
(0.090)\end{array}$ & $\begin{array}{c}0.024 \\
(0.090)\end{array}$ & $\begin{array}{c}0.027 \\
(0.090)\end{array}$ & $\begin{array}{l}0.127 \\
(0.100)\end{array}$ & $\begin{array}{l}0.120 \\
(0.100)\end{array}$ & $\begin{array}{l}0.115 \\
(0.100)\end{array}$ \\
\hline Sweden & $\begin{array}{l}0.373 * * * \\
(0.093)\end{array}$ & $\begin{array}{l}0.308^{* *} \\
(0.094)\end{array}$ & $\begin{array}{l}0.313^{* * *} \\
(0.094)\end{array}$ & $\begin{array}{l}0.395^{* * *} \\
(0.103)\end{array}$ & $\begin{array}{l}0.359 * * * \\
(0.103)\end{array}$ & $\begin{array}{l}0.360^{* * *} \\
(0.103)\end{array}$ \\
\hline Netherlands & $\begin{array}{c}0.091 \\
(0.098)\end{array}$ & $\begin{array}{c}0.031 \\
(0.098)\end{array}$ & $\begin{array}{c}0.025 \\
(0.099)\end{array}$ & $\begin{array}{c}0.153 \\
(0.104)\end{array}$ & $\begin{array}{c}0.131 \\
(0.104)\end{array}$ & $\begin{array}{c}0.117 \\
(0.104)\end{array}$ \\
\hline Spain & $\begin{array}{l}-1.117 * * * \\
(0.098)\end{array}$ & $\begin{array}{l}-1.106^{* * * *} \\
(0.098)\end{array}$ & $\begin{array}{l}-1.116^{* * *} \\
(0.099)\end{array}$ & $\begin{array}{l}-0.617 * * * \\
(0.110)\end{array}$ & $\begin{array}{l}-0.593 * * * \\
(0.110)\end{array}$ & $\begin{array}{l}-0.587 * * * \\
(0.110)\end{array}$ \\
\hline Italy & $\begin{array}{l}-0.746^{* * *} \\
(0.092)\end{array}$ & $\begin{array}{l}-0.724 * * * \\
(0.093)\end{array}$ & $\begin{array}{l}-0.731 * * * \\
(0.093)\end{array}$ & $\begin{array}{l}-0.216^{*} \\
(0.106)\end{array}$ & $\begin{array}{l}-0.185^{+} \\
(0.107)\end{array}$ & $\begin{array}{l}-0.172 \\
(0.107)\end{array}$ \\
\hline France & $\begin{array}{l}-0.315^{* * *} \\
(0.089)\end{array}$ & $\begin{array}{l}-0.359 * * * \\
(0.090)\end{array}$ & $\begin{array}{l}-0.351^{* * *} \\
(0.090)\end{array}$ & $\begin{array}{l}-0.090 \\
(0.101)\end{array}$ & $\begin{array}{l}-0.119 \\
(0.101)\end{array}$ & $\begin{array}{l}-0.114 \\
(0.101)\end{array}$ \\
\hline Denmark & $\begin{array}{r}0.244^{*} \\
(0.104)\end{array}$ & $\begin{array}{c}0.182^{+} \\
(0.105)\end{array}$ & $\begin{array}{c}0.178^{+} \\
(0.105)\end{array}$ & $\begin{array}{l}-0.068 \\
(0.119)\end{array}$ & $\begin{array}{l}-0.093 \\
(0.120)\end{array}$ & $\begin{array}{l}-0.105 \\
(0.120)\end{array}$ \\
\hline Greece & $\begin{array}{l}-0.080 \\
(0.095)\end{array}$ & $\begin{array}{l}-0.066 \\
(0.095)\end{array}$ & $\begin{array}{l}-0.074 \\
(0.095)\end{array}$ & $\begin{array}{l}0.359^{* * *} \\
(0.106)\end{array}$ & $\begin{array}{l}0.401 * * * \\
(0.106)\end{array}$ & $\begin{array}{l}0.411 * * * \\
(0.107)\end{array}$ \\
\hline Switzerland & $\begin{array}{c}0.206 \\
(0.135)\end{array}$ & $\begin{array}{c}0.180 \\
(0.1350)\end{array}$ & $\begin{array}{c}0.185 \\
(0.135)\end{array}$ & $\begin{array}{l}0.427 * * \\
(0.134)\end{array}$ & $\begin{array}{c}0.408^{* *} \\
(0.134)\end{array}$ & $\begin{array}{l}0.409 * * \\
(0.134)\end{array}$ \\
\hline Belgium & $\begin{array}{c}-0.172^{*} \\
(0.088)\end{array}$ & $\begin{array}{l}-0.202 * \\
(0.088)\end{array}$ & $\begin{array}{c}-0.213 * \\
(0.088)\end{array}$ & $\begin{array}{l}-0.010 \\
(0.094)\end{array}$ & $\begin{array}{l}-0.025 \\
(0.094)\end{array}$ & $\begin{array}{l}-0.031 \\
(0.094)\end{array}$ \\
\hline _cons & $\begin{array}{l}5.509 * * * \\
(1.432)\end{array}$ & $\begin{array}{l}5.764 * * * \\
(1.436)\end{array}$ & $\begin{array}{l}5.734 * * * \\
(1.437) \\
\end{array}$ & $\begin{array}{r}3.492 * \\
(1.670) \\
\end{array}$ & $\begin{array}{r}3.709^{*} \\
(1.680) \\
\end{array}$ & $\begin{array}{l}3.884 * \\
(1.679) \\
\end{array}$ \\
\hline $\mathrm{N}$ & 5978 & 5978 & 5978 & 4778 & 4778 & 4778 \\
\hline
\end{tabular}

Note: Standard errors in parentheses. $+\mathrm{p}<0.10,{ }^{*} \mathrm{p}<0.05,{ }^{*} \mathrm{p}<0.01,{ }^{* * *} \mathrm{p}<0.001$.

Source: SHARE Release 2.5.0, authors' calculations.

The negative coefficient of childless suggests that nulliparous women and men have lower fluency than parents (Table 3). The number of children, however, results to be related to fluency performance in a curvilinear way, especially for men: fathers of one child and fathers of four or more children name a significantly lower number of animals than fathers of two children. We found that elderly 
fathers with three children had no statistically significant lower fluency than parents of two children. Turning to recall tests, no strong association was found between number of children and recall abilities for women (Table 4 and Table 5). However, mothers of three or more children have lower immediate recall skills than mothers of two children. For men, the shape of the children-cognition relation remains concave and highlights a significant negative association between having four children or more as compared to having two and immediate and delayed recall functioning.

It may therefore be relevant to look at the relation between the parent and the child(ren) in order to draw a path for the association children-cognition. The local proximity of children results in a similar effect on women and men concerning fluency (Table 3). Parents living further away seem to have higher levels as compared to non-parents and to those living together or in close proximity with at least one child.

The results of the regressions on fluency including frequency of contact deserve to be highlighted. We detect a protective effect of contact on both mothers' and fathers' fluency (Table 3). Parent-child contact once or several times a week is significantly associated with higher levels in the fluency test. A similar direction is found for the coefficient of daily contact, although it is not statistically significant.

Geographical proximity to children as well as contact with children turns out to have little explanatory power for parents' recall abilities (Table 4 and Table 5) - with one exception: the always negative coefficient of having at least one child living in the same house(hold), which is especially significant for women's delayed recall abilities.

Concerning the other variables included in the models, a gender-specific effect of marital status emerges. Among men, the never-married result to be the most disadvantaged in terms of both fluency and immediate recall. In addition to never having married, women's recall performance seems to be also negatively affected by both voluntary and involuntary marital disruption.

Education confirms its role as the main driver of cognitive functioning. For both fluency and recall abilities, education is significantly associated with higher cognitive levels. As for the fluency test, respondents with a lower secondary degree name on average 1.3 to 1.8 more animals than their counterparts with none or just primary education. Highly educated respondents name on average more than 3 (about 3.3 for women and 3.6 for men) animals morethan low educated respondents. Concerning the recall tests, highly educated respondents recall on average 1 word more than those with little education. The indicator of vigorous physical activity shows a clear impact of behavioural contextual factors on cognitive performance in old age: engaging in sports, heavy housework or a job that involves physical labour at least once a week is associated with naming on average about one animal more. Similarly, people who engage more frequently in vigorous physical activities recall more words than those who do not. The 
biomarker included in the analyses confirms the strong association between biological factors and cognitive performance: controlling for all the other sociodemographic and contextual factors, elderly persons with higher grip strength also have higher scores in the cognitive tests.

Table 5:

Estimated coefficients from regressions for delayed recall among older women and men

\begin{tabular}{|c|c|c|c|c|c|c|}
\hline a. Explanatory variables & \multicolumn{3}{|c|}{ Women } & \multicolumn{3}{|c|}{ Men } \\
\hline \multicolumn{7}{|l|}{ Number of children (Ref. 2) } \\
\hline 0 & $\begin{array}{c}0.087 \\
(0.082)\end{array}$ & & & $\begin{array}{l}-0.080 \\
(0.094)\end{array}$ & & \\
\hline 1 child & $\begin{array}{c}0.054 \\
(0.062)\end{array}$ & & & $\begin{array}{l}-0.080 \\
(0.069)\end{array}$ & & \\
\hline 3 & $\begin{array}{l}-0.040 \\
(0.062)\end{array}$ & & & $\begin{array}{l}-0.076 \\
(0.067)\end{array}$ & & \\
\hline $4+$ & $\begin{array}{l}-0.072 \\
(0.068)\end{array}$ & & & $\begin{array}{l}-0.119^{+} \\
(0.073)\end{array}$ & & \\
\hline \multicolumn{7}{|c|}{ Location of child (Ref. 0) } \\
\hline \multicolumn{2}{|l|}{ Same house(hold) } & $\begin{array}{l}-0.239 * * \\
(0.086)\end{array}$ & & & $\begin{array}{l}-0.050 \\
(0.102)\end{array}$ & \\
\hline$<1 \mathrm{~km}$ & & $\begin{array}{l}-0.085 \\
(0.089)\end{array}$ & & & $\begin{array}{c}0.029 \\
(0.103)\end{array}$ & \\
\hline $1-25 \mathrm{~km}$ & & $\begin{array}{l}-0.057 \\
(0.082)\end{array}$ & & & $\begin{array}{c}0.044 \\
(0.094)\end{array}$ & \\
\hline $25-100 \mathrm{~km}$ & & $\begin{array}{c}0.096 \\
(0.109)\end{array}$ & & & $\begin{array}{l}-0.040 \\
(0.120)\end{array}$ & \\
\hline$>100 \mathrm{~km}$ & & $\begin{array}{c}0.003 \\
(0.114)\end{array}$ & & & $\begin{array}{c}0.183 \\
(0.123)\end{array}$ & \\
\hline \multicolumn{7}{|c|}{ Contact with child(ren) (Ref. 0) } \\
\hline \multicolumn{2}{|l|}{ Child(ren) in house(hold) } & & $\begin{array}{l}-0.231 * * \\
(0.087)\end{array}$ & & & $\begin{array}{l}-0.048 \\
(0.103)\end{array}$ \\
\hline Daily & & & $\begin{array}{l}-0.028 \\
(0.084)\end{array}$ & & & $\begin{array}{c}0.014 \\
(0.098)\end{array}$ \\
\hline \multicolumn{2}{|c|}{ Several times a week or once a week } & & $\begin{array}{l}-0.024 \\
(0.082)\end{array}$ & & & $\begin{array}{c}0.124 \\
(0.095)\end{array}$ \\
\hline Less than weekly & & & $\begin{array}{c}-0.203^{+} \\
(0.125) \\
\end{array}$ & & & $\begin{array}{l}-0.166 \\
(0.123) \\
\end{array}$ \\
\hline \multicolumn{7}{|l|}{ b. Control variables } \\
\hline \multicolumn{7}{|c|}{ Marital status (Ref. living with partner) } \\
\hline Never married & $\begin{array}{l}-0.210^{+} \\
(0.108)\end{array}$ & $\begin{array}{l}-0.201^{+} \\
(0.108)\end{array}$ & $\begin{array}{l}-0.190^{+} \\
(0.108)\end{array}$ & $\begin{array}{l}-0.009 \\
(0.126)\end{array}$ & $\begin{array}{l}-0.015 \\
(0.126)\end{array}$ & $\begin{array}{l}-0.004 \\
(0.126)\end{array}$ \\
\hline Separated / divorced & $\begin{array}{l}-0.195^{*} \\
(0.084)\end{array}$ & $\begin{array}{l}-0.194 * \\
(0.084)\end{array}$ & $\begin{array}{l}-0.181^{*} \\
(0.084)\end{array}$ & $\begin{array}{l}-0.054 \\
(0.100)\end{array}$ & $\begin{array}{l}-0.074 \\
(0.101)\end{array}$ & $\begin{array}{l}-0.028 \\
(0.102)\end{array}$ \\
\hline Widowed & $\begin{array}{l}-0.079 \\
(0.053)\end{array}$ & $\begin{array}{l}-0.065 \\
(0.053)\end{array}$ & $\begin{array}{l}-0.063 \\
(0.053)\end{array}$ & $\begin{array}{l}-0.007 \\
(0.074)\end{array}$ & $\begin{array}{l}-0.005 \\
(0.074)\end{array}$ & $\begin{array}{l}0.008 \\
(0.074)\end{array}$ \\
\hline \multicolumn{7}{|l|}{ Age } \\
\hline Age & $\begin{array}{l}-0.002 \\
(0.042)\end{array}$ & $\begin{array}{l}-0.013 \\
(0.042)\end{array}$ & $\begin{array}{l}-0.013 \\
(0.042)\end{array}$ & $\begin{array}{c}0.052 \\
(0.050)\end{array}$ & $\begin{array}{c}0.046 \\
(0.050)\end{array}$ & $\begin{array}{c}0.043 \\
(0.050)\end{array}$ \\
\hline
\end{tabular}

(Table 5 continues on next page) 


\begin{tabular}{|c|c|c|c|c|c|c|}
\hline \multirow[b]{2}{*}{ b. Control variables } & \multicolumn{3}{|c|}{ Women } & \multicolumn{3}{|c|}{ Men } \\
\hline & Model 1 & Model 2 & Model 3 & Model 1 & Model 2 & Model 3 \\
\hline Age*age/100 & $\begin{array}{l}-0.040 \\
(0.028)\end{array}$ & $\begin{array}{l}-0.033 \\
(0.028)\end{array}$ & $\begin{array}{l}-0.033 \\
(0.028)\end{array}$ & $\begin{array}{l}-0.067 * \\
(0.034)\end{array}$ & $\begin{array}{l}-0.064^{+} \\
(0.034)\end{array}$ & $\begin{array}{l}-0.062^{+} \\
(0.034)\end{array}$ \\
\hline \multicolumn{7}{|c|}{ Education (Ref. none / primary) } \\
\hline Lower secondary & $\begin{array}{l}0.417 * * * \\
(0.064)\end{array}$ & $\begin{array}{l}0.414 * * * \\
(0.064)\end{array}$ & $\begin{array}{l}0.419 * * * \\
(0.064)\end{array}$ & $\begin{array}{l}0.498 * * * \\
(0.075)\end{array}$ & $\begin{array}{l}0.499 * * * \\
(0.075)\end{array}$ & $\begin{array}{l}0.502 * * * \\
(0.075)\end{array}$ \\
\hline $\begin{array}{l}\text { Higher secondary or } \\
\text { tertiary }\end{array}$ & $\begin{array}{l}0.964 * * * \\
(0.058)\end{array}$ & $\begin{array}{l}0.952 * * * \\
(0.058)\end{array}$ & $\begin{array}{l}0.961^{* * * *} \\
(0.058)\end{array}$ & $\begin{array}{l}0.895 * * * \\
(0.060)\end{array}$ & $\begin{array}{l}0.894 * * * \\
(0.060)\end{array}$ & $\begin{array}{l}0.897 * * * \\
(0.060)\end{array}$ \\
\hline \multicolumn{7}{|c|}{ Working status and health } \\
\hline $\begin{array}{l}\text { Homemaker (Ref. } \\
\text { retired) }\end{array}$ & $\begin{array}{l}-0.101 \\
(0.160)\end{array}$ & $\begin{array}{l}-0.103 \\
(0.160)\end{array}$ & $\begin{array}{l}-0.100 \\
(0.160)\end{array}$ & $\begin{array}{l}-0.165 \\
(0.162)\end{array}$ & $\begin{array}{l}-0.151 \\
(0.162)\end{array}$ & $\begin{array}{l}-0.153 \\
(0.161)\end{array}$ \\
\hline $\begin{array}{l}\text { Permanently sick or } \\
\text { disabled }\end{array}$ & $\begin{array}{l}0.031 \\
(0.055)\end{array}$ & $\begin{array}{c}0.034 \\
(0.055)\end{array}$ & $\begin{array}{l}0.029 \\
(0.055)\end{array}$ & $\begin{array}{c}0.311 \\
(0.341)\end{array}$ & $\begin{array}{l}0.306 \\
(0.341)\end{array}$ & $\begin{array}{c}0.301 \\
(0.340)\end{array}$ \\
\hline Less than good health & $-0.224 * * *$ & $-0.218^{* * *}$ & $-0.217 * * *$ & $-0.225 * * *$ & $-0.224 * * *$ & $-0.218^{* * *}$ \\
\hline (Ref. good/very good) & $(0.049)$ & $(0.049)$ & $(0.049)$ & $(0.053)$ & $(0.053)$ & $(0.053)$ \\
\hline Euro-D depression scale & $-0.055 * * *$ & $-0.055 * * *$ & $-0.055 * * *$ & $-0.083 * * *$ & $-0.084 * * *$ & $-0.083 * * *$ \\
\hline (Ref. not depressed) & $(0.010)$ & $(0.010)$ & $(0.010)$ & $(0.013)$ & $(0.013)$ & $(0.013)$ \\
\hline $\begin{array}{l}\text { Vigorous activities } \geq 1 \text { a } \\
\text { week (Ref. less) }\end{array}$ & $\begin{array}{l}0.149 * * \\
(0.049)\end{array}$ & $\begin{array}{l}0.146^{* *} \\
(0.049)\end{array}$ & $\begin{array}{l}0.146^{* *} \\
(0.049)\end{array}$ & $\begin{array}{r}0.115^{*} \\
(0.051)\end{array}$ & $\begin{array}{r}0.113^{*} \\
(0.051)\end{array}$ & $\begin{array}{r}0.117^{*} \\
(0.051)\end{array}$ \\
\hline \multicolumn{7}{|l|}{ Biomarker } \\
\hline Grip strength (Ref. 1) & $\begin{array}{l}0.023 * * * \\
(0.004)\end{array}$ & $\begin{array}{l}0.022 * * * \\
(0.004)\end{array}$ & $\begin{array}{l}0.022 * * * \\
(0.004)\end{array}$ & $\begin{array}{l}0.012 * * * \\
(0.003)\end{array}$ & $\begin{array}{l}0.013 * * * \\
(0.003)\end{array}$ & $\begin{array}{l}0.012 * * * \\
(0.003)\end{array}$ \\
\hline \multicolumn{7}{|l|}{ Country (Ref. Austria) } \\
\hline Germany & $\begin{array}{l}-0.066 \\
(0.099)\end{array}$ & $\begin{array}{l}-0.072 \\
(0.098)\end{array}$ & $\begin{array}{l}-0.070 \\
(0.098)\end{array}$ & $\begin{array}{c}0.075 \\
(0.110)\end{array}$ & $\begin{array}{c}0.072 \\
(0.110)\end{array}$ & $\begin{array}{c}0.062 \\
(0.110)\end{array}$ \\
\hline Sweden & $\begin{array}{l}0.670 * * * \\
(0.102)\end{array}$ & $\begin{array}{l}0.607 * * * \\
(0.103)\end{array}$ & $\begin{array}{l}0.611^{* * *} \\
(0.103)\end{array}$ & $\begin{array}{l}0.468 * * * \\
(0.113)\end{array}$ & $\begin{array}{l}0.448^{* * *} \\
(0.114)\end{array}$ & $\begin{array}{l}0.430^{* * *} \\
(0.114)\end{array}$ \\
\hline Netherlands & $\begin{array}{l}0.305^{* *} \\
(0.108)\end{array}$ & $\begin{array}{c}0.248^{*} \\
(0.108)\end{array}$ & $\begin{array}{c}0.241 * \\
(0.108)\end{array}$ & $\begin{array}{c}0.163 \\
(0.115)\end{array}$ & $\begin{array}{c}0.153 \\
(0.115)\end{array}$ & $\begin{array}{c}0.129 \\
(0.115)\end{array}$ \\
\hline Spain & $\begin{array}{l}-0.613 * * * \\
(0.108)\end{array}$ & $\begin{array}{l}-0.599 * * * \\
(0.108)\end{array}$ & $\begin{array}{l}-0.608^{* * *} \\
(0.108)\end{array}$ & $\begin{array}{l}-0.364^{* *} \\
(0.121)\end{array}$ & $\begin{array}{l}-0.359^{* *} \\
(0.121)\end{array}$ & $\begin{array}{l}-0.357 * * \\
(0.122)\end{array}$ \\
\hline Italy & $\begin{array}{l}-0.538^{* * *} \\
(0.101)\end{array}$ & $\begin{array}{l}-0.510^{* * *} \\
(0.102)\end{array}$ & $\begin{array}{l}-0.516^{* * *} \\
(0.102)\end{array}$ & $\begin{array}{l}-0.159 \\
(0.117)\end{array}$ & $\begin{array}{l}-0.137 \\
(0.118)\end{array}$ & $\begin{array}{l}-0.137 \\
(0.118)\end{array}$ \\
\hline France & $\begin{array}{l}-0.072 \\
(0.098)\end{array}$ & $\begin{array}{l}-0.113 \\
(0.098)\end{array}$ & $\begin{array}{l}-0.107 \\
(0.098)\end{array}$ & $\begin{array}{l}-0.041 \\
(0.112)\end{array}$ & $\begin{array}{l}-0.059 \\
(0.112)\end{array}$ & $\begin{array}{l}-0.066 \\
(0.112)\end{array}$ \\
\hline Denmark & $\begin{array}{l}0.720^{* * *} \\
(0.114)\end{array}$ & $\begin{array}{l}0.662 * * * \\
(0.115)\end{array}$ & $\begin{array}{l}0.658^{* * *} \\
(0.115)\end{array}$ & $\begin{array}{c}0.227^{+} \\
(0.132)\end{array}$ & $\begin{array}{c}0.222^{+} \\
(0.132)\end{array}$ & $\begin{array}{c}0.194 \\
(0.132)\end{array}$ \\
\hline Greece & $\begin{array}{l}0.008 \\
(0.104)\end{array}$ & $\begin{array}{c}0.028 \\
(0.104)\end{array}$ & $\begin{array}{c}0.021 \\
(0.104)\end{array}$ & $\begin{array}{c}0.317 * * \\
(0.117)\end{array}$ & $\begin{array}{l}0.344^{* *} \\
(0.117)\end{array}$ & $\begin{array}{l}0.341 * * \\
(0.118)\end{array}$ \\
\hline Switzerland & $\begin{array}{l}0.476^{* *} \\
(0.147)\end{array}$ & $\begin{array}{l}0.450 * * \\
(0.147)\end{array}$ & $\begin{array}{l}0.456^{* *} \\
(0.147)\end{array}$ & $\begin{array}{c}0.405^{* *} \\
(0.148)\end{array}$ & $\begin{array}{l}0.398^{* *} \\
(0.148)\end{array}$ & $\begin{array}{l}0.398^{* *} \\
(0.148)\end{array}$ \\
\hline Belgium & $\begin{array}{l}-0.224 * \\
(0.096)\end{array}$ & $\begin{array}{l}-0.253^{* *} \\
(0.096)\end{array}$ & $\begin{array}{l}-0.265^{* *} \\
(0.096)\end{array}$ & $\begin{array}{l}-0.182^{+} \\
(0.103)\end{array}$ & $\begin{array}{c}-0.188^{+} \\
(0.104)\end{array}$ & $\begin{array}{c}-0.206^{*} \\
(0.104)\end{array}$ \\
\hline _cons & $\begin{array}{l}4.419 * * \\
(1.568)\end{array}$ & $\begin{array}{l}4.932 * * \\
(1.571)\end{array}$ & $\begin{array}{l}4.909 * * \\
(1.571)\end{array}$ & $\begin{array}{l}1.678 \\
(1.844)\end{array}$ & $\begin{array}{c}1.839 \\
(1.855)\end{array}$ & $\begin{array}{c}1.959 \\
(1.854)\end{array}$ \\
\hline $\mathrm{N}$ & 5978 & 5978 & 5978 & 4778 & 4778 & 4778 \\
\hline
\end{tabular}

Note: Standard errors in parentheses. $+\mathrm{p}<0.10,{ }^{*} \mathrm{p}<0.05,{ }^{*} \mathrm{p}<0.01,{ }^{* * *} \mathrm{p}<0.001$.

Source: SHARE Release 2.5.0, authors' calculations. 


\section{Discussion}

Despite the growing costs of child care, longer education, rising age at nestleaving and increasing female employment, which to a greater or less extent all contribute to the economic loss associated with high parity and to a change in the parent-child relationship, people continue to have children and tend to count on them in their later years. Whether this would imply a disadvantage for the childless may depend on the amount of welfare services offered (Dykstra and Hagestad 2007) and is a different part of the story. Yet a focus on the association between number of (adult) children and cognitive functioning of their elderly parents plays an important role in the literature and sheds light on a relevant aspect of active ageing. The primary finding of interest in this paper is the negative relation between childlessness and fluency ability and, for the parents, a positive relation between the associational dimension of intergenerational solidarity and the dimension of cognitive functioning measured with the "fluency" test in SHARE. By contrast, the results suggest that proximity to and contact with the children are not significantly correlated with cognitive performance in the form of recall functioning.

Our results on the number of children do not differ particularly from those of Grundy and Tomassini (2005) on longstanding illness and mortality or from the outcomes of Buber and Engelhardt (2008) on depression. This is consistent with research from a variety of disciplines showing that cognition and health are intertwined across the life course (see Richards and Hatch 2011 for a review). To this literature we add a more objective measure of age outcomes, encompassing health conditions in a broader meaning, and an analysis of both women and men that allows to also consider social roles and relationships other than biological explanations of the link between parity and cognition in later life.

\section{Nulliparity}

The negative coefficients for nulliparous hint at two possible explanations. In fact, on the one hand, an evolutional explanation would argue that people with low cognitive abilities are less attractive partners and, in turn, less likely to have children (e.g. Kiernan 1988; 1989). As argued by Grundy and Tomassini (2005), men and women with these characteristics may account for more of the nulliparous group in cohorts with rare nulliparity. However, selection cannot totally explain the link between nulliparity and cognition as such an association should hold for all the dimensions of cognitive functioning considered. Moreover, post-reproductive differences are not subject to selection (e.g. Comfort 1956). On the other hand, identifying cognitive functioning as an outcome of contextual influences would suggest that elderly people without children may lack social support and are less likely to be involved in activities which could help prevent the age-related cognitive decline: for example, they are not engaged in grandparenting which could, per se, stimulate cognitive abilities (e.g. Arpino and 
Bordone 2012) and therefore improve health of the elderly generation. Gruenewald et al. (2007) find a strong positive association between feeling useful and health in older ages. Supportive ties with children are indeed an important link between total family size and cognitive functioning at older ages.

\section{One child}

Poorer outcomes in fluency were also observed for parents with only one child. Again both biological and contextual effects may be important. Those with only one child will include people with health problems, and in the case of women also problems related to the first pregnancy which prevented or discouraged them from having more children (see Grundy and Tomassini 2005 for a discussion); however, the significant result for men, pointing to the same direction of association, suggests that lower levels of social support may be a fact for those with one child, as well as for the childless: parents who have only one child are, for example, less likely to see their child at least once a week than parents with two or more children (Tomassini et al. 2004).

\section{Higher parities}

Concerning the negative association between number of children and cognitive functioning, the explanation for women may lie in the association between high parities and short birth intervals. ${ }^{4}$ Ample literature (mainly focusing on less developed countries) on links between fertility and maternal health has shed light on the adverse consequences of short birth intervals for mothers. However, the statistically significant findings for men, pointing to the same direction of the effect, further support the notion of a non-biological pathway linking family size and poorer cognitive functioning in later life. This hints once more at interpreting the number of children as an indicator for the quantity of support to the parents (see Buber and Engelhardt 2008). According to the strand of literature started by Wheaton's question, "Can there be too much of a good thing"? (1985), high support from the children may reduce the sense of control of the parents. The affective, behavioural, motivational and physiological effects of a lowered sense of control may in turn decrease cognitive performance by increasing levels of stress and anxiety, decreasing levels of effort, as well as reducing frequent engagement in memory tasks (e.g. Miller and Lachman 2000; West and Yassuda 2004).

In order to further explain the curvilinear association of number of children and cognition, Table A.1 in the Appendix shows the characteristics of the study's sub-samples by number of children $(0,1,2,3$ and $4+)$ : parents of two or three children are more likely (than parents of a single child or of four children and

\footnotetext{
Evidence from the sample used in this study confirms that respondents with higher parity also had shorter birth intervals.
} 
more) to live with a partner, to have high education (especially fathers), to live only a few kilometres away from the closest child (but not in the same household) and to maintain a high frequency of contact with the child(ren). The current study cannot test the reasons behind this selection. However, one line of argumentation would argue that two children are about the 'standard' or the 'norm', referring to individual preferences (see e.g. Lutz and Scherbov (2008) for a discussion): men and women may naturally desire to replace themselves and therefore have two children rather than one or three (under low-mortality conditions). Other characteristics would play a role in the 'exceptions' (i.e. one child and four children or more).

\section{Geographical proximity and frequency of contact}

As with higher parities, having at least one child living in the same house(hold) is found to be negatively associated with cognitive abilities. Closer involvement with children may imply more conflict (e.g. mothers, who are usually closer to children than the fathers, also tend to experience more conflictual relationships with them (Fischer 1982; Gerstel and Gallagher 1993)) and predispose parents to the so-called "contagion of stress": when stressful events afflict the child, the parents face the related (emotional) costs together with the child (Belle 1982). However, we acknowledge that this result might also reflect the causal relationship between parents' cognitive decline and moving together with or close to a child (as argued by Buber and Engelhardt 2008).

Furthermore, the results suggest that fewer contacts with children were associated with lower fluency abilities, in line with the results on depression found by Buber and Engelhardt (2008). Although contact does not always correspond with the quality of the parent-child relationships, it may influence cognitive functioning in several ways: a greater sense of purpose could have direct neurohormonal benefits (Fratiglioni et al. 2004); while contact with children may also serve as a remainder to take care of oneself. As Buber and Engelhardt have argued (2008, p. 42), "very little contact with one's children would normally be interpreted as a [...] lack of concern for one's old parents". By contrast, we might also think that parents who have better fluency performance might initiate contact with children more often or be a more interesting partner of conversation.

\section{The control variables and gender differences}

The outcomes of the control variables are in line with previous research and we therefore do not discuss them in detail. However, of particular interest are the education effects, which remain large and significant even after controlling for socio-demographic and child-related characteristics. The coefficient for four or more children suggests a negative association with fluency for men though, which reduces by more than one-third the positive association with having a lower 
secondary rather than a primary education (that is about 1.8 words). In turn, however, lower secondary education more than counterbalances the negative (additive) association between poor health (disability, less-than-good selfreported health, depression) and recall tests.

Gender-specific behaviours within the societies considered may affect the gender gap in these results. We follow the literature on the larger effect of marriage on men's health that focuses on biological differences, socio-economic roles, health-reporting behaviour (Verbrugge 1985; Macintyre et al. 1996), but also on the fact that women are more socialised to go to medical doctors (Johnson-Lans and Bellemore 1997) and engage more often in social activities as compared to men (e.g. Agahi et al. 2011). Since all these behaviours have been shown to help maintain cognitive abilities over time, women are less in need of the presence of a controller to keep their cognitive functioning. By contrast, confirming the results of Kendig and colleagues (2007), men are more likely to be affected by the presence (or absence) of children: in the same way as men need 'guardian wives', they may also need to commit to children in order to adopt a healthy lifestyle and abandon what has been called "stupid bachelor tricks" (Umberson 1992). However, men's mobilisation of support is heavily focused on their spouses (Lowenthal and Haven 1968), whereas women usually receive more help from their children (Fischer 1982; Rossi and Rossi 1990; Silverstein et al. 2006). Therefore, fathers are more likely to be exposed to a potential loss of their sense of purpose and a consequent decline in cognitive abilities when faced with large amounts of assistance from the younger generation in later life, perceiving the role reversal as undermining the balance between autonomy and dependency (Bengtson and Kuypers 1986). Further studies may additionally distinguish between daughters and sons in order to account for different types of interaction according to the gender of children. Moreover, the gender composition of the children may be considered as an influence on family relationships independent of the child's own characteristics.

\section{Conclusive remarks}

It has been theorised that much of the cognitive decline with age can be traced back to deficits in the information processing system. However, most of the studies on social interaction and cognition have focused on only one dimension of cognitive performance (e.g. Engelhardt et al. (2010) examined only the recall task). This study, which considers three tests of cognitive performance, confirms that different aspects of cognition are modifiable in different ways. Tasks that use well-practiced skills or familiar information, such as those tested with the fluency test, are recognised to be more amenable to change as they rely on specific acquired knowledge. Generally, these abilities have a less pronounced decline with age. The presence and interaction with children results to be more strongly 
associated with this performance. By contrast, children and intergenerational solidarity seem to be less able to counteract the cognitive decline in tasks that require taking in new information, such as the abilities tested in the two recall tests. Indeed, recall tasks involve components which show a more pronounced decline with age and may therefore leave less room to changes (Verhaegen and Salthouse 1997; Deary et al. 2010). However, knowledge does not only play a critical role in activities that involve learning new information but in all cognitive tasks (Hertzog 2008). The different results for fluency and recall may also suggest that the content of interaction is important. Unfortunately SHARE does not provide information about the nature of activities engaged in by parents and children during the time spent together. This would add to the understanding of different associations between intergenerational relationships and cognitive functioning.

We acknowledge that the obtained results should be considered in light of their limitations. The findings presented in this article document a suggestive association between children and later-life fluency functioning. The analyses do not necessarily reveal a causal relationship between these measures and thus need to be interpreted with some caution. However, the cross-sectional approach overcomes the positive selection of the longitudinal participants in SHARE (Zamarro et al. 2008) as well as the retest effects which would affect cognitive tests (Schaie and Hofer 2001; Mazzonna and Peracchi 2012). Moreover, relatively few studies on the relation between number of children and health-related aspects in later life have included men in their sample. By doing this, we are able to consider both biological mechanisms and social relationships linking parity and cognitive functioning in later life. Methodologically, disaggregating the groups childless, parity one, two, three and higher parities allows a better understanding of the interlinkages between reproductive history and longevity. Future studies may consider using an instrumental variables approach or structural equation modelling in order to obtain further hints about the direction of causality in the association between number of children and cognitive functioning.

Along with the factors we considered in the current study, other variables may play a role in cognitive functioning as well. Risk factors (such as being overweight, smoking and having alcohol problems) are likely to reduce cognitive functioning, just as health factors (such as physical exercise considered here) help maintain good cognitive performance. Furthermore, on the one hand, level of stress and personality profiles also account for between-person heterogeneity in cognitive performance, directly or via physical health (see Agrigoroaei and Lachman 2011 for a review). On the other hand, although explaining the crosscountry divide in average levels of cognitive abilities goes beyond the goal of this study, cultural differences may also be relevant in a cross-country comparative analysis: education system, retirement patterns, as well as attitude and life style are among the several aspects that future research should consider (Skirbekk et al. 2012). 
In spite of these limitations, the present study is a plausible examination of the association between number of children and cognitive functioning in old age. Our analysis supports the idea that individual performance depends on both personal attributes and contextual factors (as suggested by Hertzog et al. 2009). One final note about possible social implications of these findings: although this study reports on number of children, an aspect over which individuals have some degree of control, we caution against influencing fertility choices on the basis of these findings. Follow-up studies are required to confirm the effects of number of, and relationship with, children on cognitive decline over the life course.

Since frequent contacts with the children result in a higher awareness of each other's needs, that in turn improves the perceived quality of the relationships (Kalmijn and Dykstra 2006), we can conclude, however, that a supportive relationship with their children is an important resource in offsetting cognitive ageing for both women and men. Data distinguishing between phone calls and face-to-face contact may also additionally help disentangle the protective effect of various dimensions of support.

\section{Acknowledgments}

The authors are thankful to Carolina E. Küpper-Tetzel from the Department of Psychology of the University of Mannheim for her precious help in defining and interpreting cognitive measures.

This research was funded by the Austrian Science Fund (FWF): Z171-G11 and by an Advanced Grant of the European Research Council, ERC Grant Agreement No. 241003-COHORT.

This paper uses data from SHARE release 2.5.0, as of May 24th 2011. The SHARE data collection has been primarily funded by the European Commission through the 5th framework programme (project QLK6-CT-2001-00360 in the thematic programme Quality of Life), through the 6th framework programme (projects SHARE-I3, RII-CT-2006-062193, COMPARE, CIT5-CT-2005-028857, and SHARELIFE, CIT4-CT-2006-028812) and through the 7th framework programme (SHARE-PREP, 211909 and SHARE-LEAP, 227822). Additional funding from the U.S. National Institute on Aging (U01 AG09740-13S2, P01 AG005842, P01 AG08291, P30 AG12815, Y1-AG-4553-01 and OGHA 04-064, IAG BSR06-11, R21 AG025169) as well as from various national sources is gratefully acknowledged (see www.share-project.org for a full list of funding institutions). 


\section{References}

Agahi, N., M. Silverstein and M.G. Parker. 2011. "Late-life and earlier participation in leisure activities: their importance for survival among older persons." Activities, Adaptation \& Aging 35(3): 210-222.

Agrigoroaei, S. and M.E. Lachman. 2011. "Cognitive functioning in midlife and old age: combined effects of psychosocial and behavioral factors." The Journals of Gerontology, Series B: Psychological Sciences and Social Sciences 66B(S1): i130i140.

Alley, D., K. Suthers and E. Crimmins. 2007. "Education and cognitive decline in older Americans: results from the AHEAD sample." Research on Aging 29: 73-94.

Anstey, K.J., Lord, S.R., and Smith, G.A. 1996. Human functional age research: A review of empirical findings. Experimental Aging Research 22(3):245-266.

Anstey, K.J. and G. Smith. 1999. "Interrelationships among biological markers of aging, health, activity, acculturation, and cognitive performance in late adulthood." Psychology and Aging 14(4): 605-618.

Antonucci, T.C. 1990. "Social supports and social relationships." In Handbook of Aging and the Social Sciences, ed. R.H. Binstock and L.K. George, 205-226. San Diego, CA: Academic Press.

Arpino, B. and V. Bordone. 2012. "Does grandparenting pay off? The effect of childcare on grandparents' cognitive functioning." European Demographic Research Papers 4. Vienna: Vienna Institute of Demography of the Austrian Academy of Sciences.

Balin, A.K. 1994. Practical handbook of biologic age determination. Boca Raton, FL: CRC Press.

Ball, K., J.D. Edwards and L.A. Ross. 2007. "The impact of speed of processing training on cognitive and everyday functions." The Journals of Gerontology, Series B: Psychological Sciences and Social Sciences 62B(Special Issue I): 19-31.

Baltes, P.B. 1987. "Theoretical propositions of life-span developmental psychology: On dynamics between growth and decline." Developmental Psychology 23(5): 611-626.

Baltes, P.B. and G.V. Labouvie. 1973. "Adult development of intellectual performance: Description, explanation, and modification." In The Psychology of Adult Development and Aging, ed. C. Eisdorfer and M.P. Lawton, 157-219. Washington, DC: American Psychological Association.

Baltes, P.B. and U. Lindenberger. 1988. "On the range of cognitive plasticity in old age as a function of experience: 15 years of intervention research." Behavior Therapy 19: 283-300.

Baltes, P.B., U. Lindenberger and U.M. Staudinger. 2006. "Lifespan theory in developmental psychology." In Handbook of Child Psychology, ed. W. Damon and R.M. Lerner, 569-664. New York: Wiley.

Bassuk, S., T. Glass and L. Berkman. 1999. "Social disengagement and incident cognitive decline in community-dwelling elderly persons." Annals of Internal Medicine 131(3): 165-173.

Batty, G.D., I.J. Deary, I. Schoon and C.R. Gale. 2007. "Mental ability across childhood in relation to risk factors for premature mortality in adult life: the 1970 British Cohort Study." Journal of Epidemiology and Community Health 61(11): 997-1003. 
Béland, F., M.-V. Zunzunegui, B. Alvarado, A. Otero and T. del Ser. 2005. "Trajectories of cognitive decline and social relations." The Journals of Gerontology, Series B: Psychological Sciences and Social Sciences 60(6): 320-330.

Belle, D. 1982. Lives in Stress: Women and Depression. Beverly Hills, CA: Sage.

Bengtson, V.L. and J.A. Kuypers. 1986. "The family support cycle: Psycho-social issues in the aging family." In Life span and change in a gerontological perspective, ed. J.M.A. Munnichs, P. Mussen and E. Olbrich, 61-77. New York: Academic Press.

Bengtson, V.L. and R.E.L. Roberts. 1991. "Intergenerational solidarity in aging families: an example of formal theory construction." Journal of Marriage and Family 53(4): 856-870.

Berkman, L.F., T. Glass, I. Brissette and T.E. Seeman. 2000. "From social integration to health: Durkheim in the new millennium." Social Science\& Medicine 51(6): 843-857.

Bonsang, E., S. Adam and S. Perelman. 2012. "Does retirement affect cognitive functioning?" Journal of Health Economics 31(3): 490-501.

Börsch-Supan, A., A. Brugiavini, H. Jürges, J. Mackenbach, J. Siegrist and G. Weber, eds. 2005. Health, ageing and retirement in Europe - First results from the Survey of Health, Ageing and Retirement in Europe. Mannheim: Mannheim Research Institute for the Economics of Aging (MEA).

Buber, I. and H. Engelhardt. 2008. "Children's impact on the mental health of their older mothers and fathers: findings from the Survey of Health, Ageing and Retirement in Europe." European Journal of Ageing 5(1): 31-45.

Cagney, K.A. and D.S. Lauderdale. 2002. "Education, wealth, and cognitive function in later life." Journal of Gerontology, Series B: Psychological Sciences and Social Sciences 57(2): P163-P172.

Cattell, R.B. 1943. "The measurement of adult intelligence." Psychological Bulletin 40(3): 153-193.

Chaves, L.M., A.L. Camozzato, C. Laks Eizirik and J. Kaye. 2009. "Predictors of normal and successful aging among urban-dwelling elderly Brazilians." Journal of Gerontology, Series B: Psychological Sciences and Social Sciences 64B(5): 597-602.

Christensen, H., A. Korten, A. Jorm, A. Henderson, R. Scott and A. MacKinnon. 1996. "Activity levels and cognitive functioning in an elderly community sample." Age and Ageing 25(1): 72-80.

Comfort, A. 1956. The Biology of Senescence. London: Routledge \& Kegan Paul.

Deary, I.J., S.E. Harris, H.C. Fox, C. Hayward, A.F. Wright, J.M. Starr and L.J. Whalley. 2005. "KLOTHO genotype and cognitive ability in childhood and old age in the same individuals." Neuroscience Letters 378(1): 22-27.

Deary, I.J.P., L. Penke and W. Johnson. 2010. "The neuroscience of human intelligence differences." Nature Reviews Neuroscience 11: 201-211.

Doblhammer, G. 2000. "Reproductive history and mortality later in life: a comparative study of England and Wales and Austria." Population Studies 54(2): 169-176.

Doblhammer, G. and J. Oeppen. 2003. "Reproduction and longevity among the British peerage: the effect of frailty and health selection." Proceedings of the Royal Society B: Biological Sciences 270(1524): 1541-1547.

Dykstra, P.A. and G.O. Hagestad. 2007. "Childlessness and parenthood in two centuries: different roads - different maps?” Journal of Family Issues 28(11): 1518-1532.

Engelhardt, H., I. Buber-Ennser, V. Skirbekk and A. Fürnkranz-Prskawetz. 2010. "Social engagement, behavioural risks and cognitive functioning among the aged." Ageing \& Society 30(5): 779-809. 
Emery, C.F., R.L. Schein, E.R. Hauck and N.R. MacIntyre. 1998. "Psychological and cognitive outcomes of a randomized trial of exercise among patients with chronic obstructive pulmonary disease." Health Psychology 17(3): 232-240.

Fabrigoule, C., L. Letenneur, J. Dartigues, M. Zarrouk, D. Commenges and P. BarbergerGateau. 1995. "Social and leisure activities and risk of dementia: a prospective longitudinal study." Journal of the American Geriatrics Society 43(5): 485-490.

Finkel, D., R. Andel, M. Gatz and N.L. Pedersen. 2009. "The role of occupational complexity in trajectories of cognitive aging before and after retirement." Psychology and Aging 24(3): 563-573.

Fischer, C.S. 1982. To Dwell Among Friends: Personal Networks in Town and City. Chicago: University of Chicago Press.

Fratiglioni, L., S. Paillard-Borg and B. Winblad. 2004. "An active and socially integrated lifestyle in late life might protect against dementia." Lancet Neurology 3(6): 343-353.

Fratiglioni, L., H. Wang, K. Ericsson, M. Maytan and B. Winblad. 2000. "The influence of social network on the occurrence of dementia: a community-based longitudinal study." Lancet 355(9212): 1315-1319.

Gerstel, N. and S.K. Gallagher. 1993. "Kin keeping and distress: Gender, recipients of care, and work-family conflict." Journal of Marriage and Family 55(3): 598-607.

Glisky, E.L., M.R. Polster and B.C. Routhieaux. 1995. "Double dissociation between item and source memory." Neuropsychology 9(2): 229-235.

Gruenewald, T., A. Karlamangla, G. Greendale, et al. 2007. "Feelings of usefulness to others, disability, and mortality in older adults: the MacArthur Study of Successful Aging." The Journals of Gerontology, Series B: Psychological Sciences and Social Sciences 62(1): P28-P37.

Grundy, E. and G. Holt. 2000. "Adult life experiences and health in early old age in Great Britain." Social Science \& Medicine 51(7): 1061-1074.

Grundy, E. and C. Tomassini. 2005. "Fertility history and health in later life: a record linkage study in England and Wales." Social Science \& Medicine 61(1): 217-228.

Grundy, E. and O. Kravdal. 2008. "Reproductive history and mortality in late middle age among Norwegian men and women." American Journal of Epidemiology 167(3): 271-279.

Gwozdz, W. and A. Sousa-Poza. 2010. "Ageing, health and life satisfaction of the oldest old: an analysis for Germany." Social Indicators Research 97(3): 397-417.

Hauser, R.M. and C.L. Roan. 2007. "Work complexity and cognitive functioning at midlife: cross-validating the Kohn-Schooler hypothesis in an American cohort." $C D E$ Working Paper 2007-08. Madison, WI: University of Wisconsin, Center for Demography and Ecology.

Hertzog, C. 2008. "Theoretical approaches to the study of cognitive aging: An individual differences perspective." In Handbook of Cognitive Aging, ed. S.M. Hofer and D.F. Alwin, 34-49. Thousand Oaks, CA: Sage Publications.

Hertzog, C., A.F. Kramer, R.S. Wilson and U. Lindenberger. 2009. "Enrichment effects on adult cognitive development." Psychological Science in the Public Interest 9(1): $1-65$.

Johnson-Lans, S. and F. Bellemore. 1997. "Gender and race as factors in health care utilization." International Advances in Economic Research 3(2): 193-205.

Jopp, D. and C. Hertzog. 2007. "Activities, self-referent memory beliefs, and cognitive performance: Evidence for direct and mediated effects." Psychology and Aging 22(4): 811-825. 
Kalmijn, M. and P. Dykstra. 2006. "Differentials in face-to-face contact between parents and their grown-up children." In Family Solidarity in the Netherlands, ed. P.A. Dykstra, M. Kalmijn, T.C.M. Knijn, A.E. Komter, A.C. Liefbroer and C.H. Mulder, 63-88. Amsterdam: Dutch University Press.

Kendig, H., P.A. Dykstra, R.I. van Gaalen and T. Melkas. 2007. „Health of aging parents and childless individuals." Journal of Family Issues 28(11): 1457-1486.

Kiernan, K. 1988. "Who remains celibate?” Journal of Biosocial Science 20(3): 253-263.

Kiernan, K. 1989. "Who remains childless?” Journal of Biosocial Science 21(4): 387-398.

Kirkwood, T. and M. Rose. 1991. "Evolution of senescence: late survival sacrificed for reproduction.” Philosophical Transactions: Biological Sciences 332(1262): 15-24.

Kramer, A.F. and S.L. Willis. 2002. "Enhancing the cognitive vitality of older adults." Current Directions in Psychological Science 11(5): 173-177.

Kramer, A.F. and S.L. Willis. 2003. "Cognitive plasticity and aging." In The Psychology of Learning and Motivation, ed. B.H. Ross, 267-302. San Diego, CA: Academic Press.

Le Carret, N., S. Laffont, W. Mayo and C. Fabrigoule. 2003. "The effect of education on cognitive performances and its implication for the constitution of the cognitive reserve." Developmental Neuropsychology 23(3): 317-337.

Lee, S., I. Kawachi, L.F. Berkman and F. Grodstein. 2003. "Education, other socioeconomic indicators, and cognitive function." American Journal of Epidemiology 157(8): 712-720.

Lowenthal, M.J. and C. Haven. 1968. "Interaction and adaptation: intimacy as a critical variable." American Sociological Review 33(1): 20-30.

Luszcz, M., J. Bryan and P. Kent. 1997. "Predicting episodic memory performance of very old men and women: Contribution from age, depression, activity, cognitive ability, and speed." Psychology and Aging 12(2): 340-351.

Lutz, W. and S. Scherbov. 2008. "Exploratory extension of IIASA's population projections: scenarios to 2300." IIASA Interim Report IR-08-022. International Institute for Applied Systems Analysis: Laxenburg, Austria.

Macintyre, S., K. Hunt and H. Sweeting. 1996. "Gender differences in health: are things really as simple as they seem?" Social Science and Medicine 42(4): 617-624.

Mazzonna, F. and F. Peracchi. 2012. "Ageing, cognitive abilities and retirement." European Economic Review 56(4): 691-710.

McLay, R.N., P.M. Maki and C.G. Lyketsos. 2003. "Nulliparity and late menopause are associated with decreased cognitive decline." The Journal of Neuropsychiatry and Clinical Neurosciences 15(2): 161-167.

Miller, L.M.S. and M.E. Lachman. 2000. "Cognitive performance and the role of control beliefs in midlife." Aging, Neuropsychology, and Cognition 7(2): 69-85.

Nappi, R.E, E. Sinforiani, M. Mauri, et al. 1999. "Memory functioning at menopause: impact of age in ovariectomized women." Gynecologic and Obstetric Investigation 47(1): 29-36.

Oberauer, K., H.-M. Suess, R. Schulze, O. Wilhelm and W.W. Wittmann. 2000. "Working memory capacity - facets of a cognitive ability construct." Personality and Individual Differences 29(6): 1017-1045.

O’Donovan, M.C. and M.J. Owen. 2009. "Genetics and the brain: many pathways to enlightenment." Human Genetics 126(1): 1-2. 
Pedersen, N.L., R. Plomin, J. Nesselroade and G.E. McClearn. 1992. "A quantitative genetic analysis of cognitive abilities during the second half of the life span." Psychological Science 3 (6): 346-352.

Richards, M. and S.L. Hatch. 2011. "A life course approach to the development of mental skills." The Journals of Gerontology, Series B: Psychological Sciences and Social Sciences 66B(suppl 1): i26-i35.

Rossi, A.S. and P.H. Rossi. 1990. Of Human Bonding, Parent-Child Relations Across the Life Course. New York: Aldine de Gruyter.

Salthouse, T.A. 2010. "Selective review of cognitive aging." Journal of the International Neuropsychological Society 16 (5): 754-760.

Scarmeas, N. and Y. Stern. 2003. "Cognitive reserve and lifestyle." Journal of Clinical \& Experimental Neuropsychology 25(5): 625-663.

Schaie, K.W. and S.M. Hofer. 2001. "Longitudinal studies in research on aging." In Handbook of the Psychology of Aging, ed. J.E. Birren and K.W. Schaie, 53-77. San Diego, CA: Academic Press.

Seeman, T., T. Lusignolo, M. Albert and L. Berkman. 2001. "Social relationships, social support, and patterns of cognitive aging in healthy, high-functioning older adults: MacArthur Studies of Successful Aging." Health Psychology 20(4): 243-255.

Silverstein, M., X. Chen and K. Heller. 1996. "Too much of a good thing? Intergenerational social support and the psychological well-being of older parents." Journal of Marriage and Family 58(4): 970-982.

Silverstein, M., D. Gans, D. and F.M. Yang. 2006. "Intergenerational support to aging parents: the role of norms and needs." Journal of Family Issues 27(8): 1068-1084.

Skirbekk, V. 2008. "Fertility trends by social status." Demographic Research 18(5): 145-180.

Skirbekk, V., D. Weber and V. Bordone. 2012. "National variation in cognitive life cycle development." IIASA Interim Report IR-11-028. International Institute for Applied Systems Analysis: Laxenburg, Austria.

Smith, C.A., C.A. McCleary, G.A. Murdock, et al. 1999. "Lifelong estrogen exposure and cognitive performance in elderly women." Brain and Cognition 39(3): 203-218.

Spiro, A. and C.B. Brady. 2008. "Integrating health into cognitive aging research and theory: quo vadis?" In Handbook of Cognitive Aging: Interdisciplinary Perspectives, ed. S.M. Hofer and D.F. Alwin, 260-283. Thousand Oaks, CA: Sage Publications.

Tomassini, C., S. Kalogirou, E. Grundy, T. Fokkema, P. Martikainen, M. Broese van Groenou and A. Karisto. 2004. "Contacts between elderly parents and their children in four European countries: current patterns and future prospects." European Journal of Ageing 1(1): 54-63.

Tuong Nguyen, C., M.-C. Couture, B. Alvarado and M. Zunzunegui. 2008. "Life course socioeconomic disadvantage and cognitive function among the elderly population of seven capitals in Latin America and the Caribbean." Journal of Aging and Health 20(3): 347-362.

Umberson, D. 1992. "Gender, marital status, and the social control of health behavior." Social Science and Medicine 34(8): 907-917.

Verbrugge, L. 1985. "Gender and health: an update on hypotheses and evidence." Journal of Health and Social Behavior 26(3): 156-182.

Verhaegen, P. and T.A. Salthouse. 1997. "Meta-analyses of age cognition relations in adulthood. Estimates of linear and nonlinear age effects and structural models." Psychological Bulletin 122(3): 231-249. 
West, R.L. and M.S. Yassuda. 2004. "Aging and memory control beliefs: performance in relation to goal setting and memory self-evaluation." The Journals of Gerontology, Series B: Psychological Sciences and Social Sciences 59(2): P56-P65.

Wethington, E., J.D. McLeod and R.C. Kessler. 1987. "The importance of life events for explaining sex differences in psychological distress." In Gender and Stress, ed. R.C. Barnett, L. Biener, and G.K. Baruch, 144-156. New York: The Free Press.

Whalley, L.J. and I.J. Deary. 2001. "Longitudinal cohort study of childhood IQ and survival up to age 76." British Medical Journal 322(7290): 819-822.

Wheaton, B. 1985. "Personal resources and mental health: can there be too much of a good thing?" In Research in Community and Mental Health, ed. J.R. Greenley, 139-184. Greenwich, CT: Jai Press.

Willis, S.L., S.L. Tennstedt, M. Marsiske, K. Ball, J. Elias, K.M. Koepke, et al. 2006. "Long-term effects of cognitive training on everyday functional outcomes in older adults." Journal of the American Medical Association 296(23): 2805-2814.

Yount, K.M. 2008. "Gender, resources across the life course, and cognitive functioning in Egypt.” Demography 45(4): 907-926.

Zamarro, G., E. Meijer and M. Fernandes. 2008. "Mental health and cognitive ability. In First results from the Survey of Health, Ageing and Retirement in Europe (20042007). Starting the longitudinal dimension, ed. A. Börsch-Supan, A. Brugiavini, H. Jürges, A. Kapteyn, et al., 40-47. Mannheim: Mannheim Research Institute for the Economics of Aging (MEA). 


\section{Appendix}

Table A.1:

Distribution of variables (\%) for men and women, by number of children

\begin{tabular}{|c|c|c|c|c|c|c|c|c|c|c|}
\hline \multirow[b]{2}{*}{ Numb of children } & \multicolumn{5}{|c|}{ Women } & \multicolumn{5}{|c|}{ Men } \\
\hline & $\mathbf{0}$ & 1 & 2 & 3 & $4+$ & $\mathbf{0}$ & 1 & 2 & 3 & $4+$ \\
\hline \multicolumn{11}{|l|}{ Marital status } \\
\hline Living with partner & 19.2 & 36.6 & 46.0 & 47.3 & 41.8 & 38.9 & 74.7 & 81.4 & 82.4 & 78.5 \\
\hline Never married & 43.0 & 3.0 & 0.6 & 0.4 & 0.8 & 43.8 & 1.0 & 0.5 & 0.2 & 0.4 \\
\hline Separated / divorced & 6.4 & 11.2 & 9.0 & 8.0 & 6.1 & 7.1 & 7.9 & 5.7 & 4.9 & 5.7 \\
\hline Widowed & 31.5 & 49.2 & 44.4 & 44.3 & 51.4 & 10.2 & 16.4 & 12.4 & 12.5 & 15.5 \\
\hline \multicolumn{11}{|l|}{ Age } \\
\hline Mean age & 73.8 & 73.0 & 71.4 & 71.6 & 73.0 & 71.7 & 71.4 & 70.9 & 71.5 & 72.8 \\
\hline \multicolumn{11}{|l|}{ Education } \\
\hline None / primary & 40.4 & 44.7 & 48.1 & 52.2 & 59.1 & 41.7 & 34.7 & 35.1 & 37.7 & 48.5 \\
\hline Lower secondary & 19.3 & 19.4 & 19.4 & 18.6 & 19.9 & 14.2 & 16.0 & 15.4 & 14.3 & 15.6 \\
\hline Higher sec. or tert. & 40.4 & 35.9 & 32.5 & 29.2 & 21.0 & 44.2 & 49.3 & 49.5 & 48.0 & 35.9 \\
\hline \multicolumn{11}{|c|}{ Working status and health } \\
\hline Retired & 80.1 & 76.0 & 68.7 & 64.0 & 60.5 & 96.4 & 97.6 & 96.8 & 97.7 & 97.3 \\
\hline Homemaker & 17.1 & 22.4 & 29.7 & 33.3 & 37.8 & 0.5 & 0.5 & 0.4 & 0.7 & 0.5 \\
\hline $\begin{array}{l}\text { Permanently sick or } \\
\text { disabled }\end{array}$ & 2.8 & 1.6 & 1.6 & 2.7 & 1.7 & 3.1 & 2.0 & 2.8 & 1.6 & 2.2 \\
\hline Less than good health & 51.3 & 55.4 & 47.2 & 49.5 & 52.9 & 41.5 & 42.0 & 40.9 & 41.0 & 49.7 \\
\hline Mean depression & 3.0 & 3.0 & 2.8 & 2.9 & 3.1 & 2.1 & 1.9 & 1.8 & 1.9 & 2.2 \\
\hline $\begin{array}{l}\text { Vigorous activities } \\
\geq 1 \text { week }\end{array}$ & 29.8 & 32.2 & 38.0 & 388 & 29.8 & 37.1 & 44.9 & 45.9 & 46.6 & 37.1 \\
\hline \multicolumn{11}{|l|}{ Biomarker } \\
\hline Mean grip strength & 23.1 & 23.5 & 24.0 & 24.0 & 23.2 & 37.8 & 39.6 & 40.0 & 40.0 & 38.0 \\
\hline \multicolumn{11}{|c|}{ Location of child living closest } \\
\hline Child(ren) in house(hol & & 22.7 & 25.1 & 27.6 & 32.7 & & 18.7 & 21.8 & 23.3 & 30.2 \\
\hline$<1 \mathrm{~km}$ & & 13.7 & 20.0 & 23.1 & 25.4 & & 14.2 & 20.0 & 22.3 & 23.6 \\
\hline $1-25 \mathrm{~km}$ & & 38.3 & 39.8 & 39.2 & 34.5 & & 39.6 & 41.1 & 41.5 & 36.3 \\
\hline $25-100 \mathrm{~km}$ & & 11.7 & 8.3 & 7.0 & 4.5 & & 11.4 & 9.4 & 8.0 & 6.0 \\
\hline$>100 \mathrm{~km}$ & & 13.6 & 6.8 & 3.1 & 2.6 & & 16.2 & 7.7 & 5.4 & 3.9 \\
\hline \multicolumn{11}{|l|}{ Contact with child(ren) } \\
\hline Daily & & 26.3 & 31.2 & 32.5 & 29.8 & & 25.0 & 29.7 & 31.1 & 27.8 \\
\hline Once or several times a & veek & 41.1 & 39.4 & 36.9 & 34.4 & & 40.7 & 41.9 & 40.5 & 35.2 \\
\hline Less than weekly & & 9.9 & 4.4 & 3.0 & 3.1 & & 15.6 & 6.5 & 5.1 & 6.8 \\
\hline $\mathrm{N}$ & 835 & 1160 & 1934 & 1140 & 909 & 607 & 819 & 1698 & 916 & 738 \\
\hline
\end{tabular}

Source: SHARE Release 2.5.0, authors' calculations. 